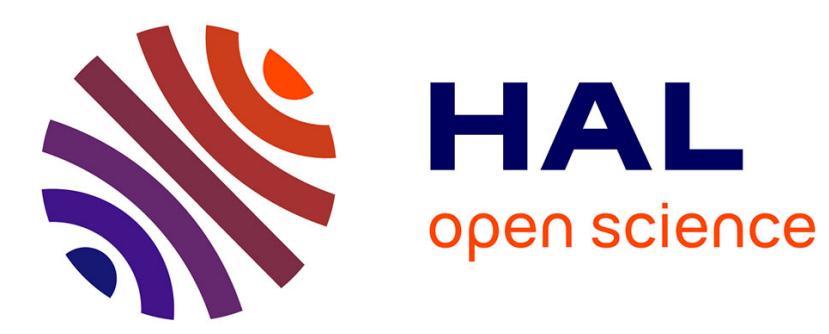

\title{
Direct numerical simulation of a reacting turbulent channel flow with thermochemical ablation
}

Olivier Cabrit, Franck Nicoud

\section{To cite this version:}

Olivier Cabrit, Franck Nicoud. Direct numerical simulation of a reacting turbulent channel flow with thermochemical ablation. Journal of Turbulence, 2010, 11 (44), pp.1-33 PII 928440181. 10.1080/14685248.2010.522578 . hal-00803379

\section{HAL Id: hal-00803379 \\ https://hal.science/hal-00803379}

Submitted on 21 Mar 2013

HAL is a multi-disciplinary open access archive for the deposit and dissemination of scientific research documents, whether they are published or not. The documents may come from teaching and research institutions in France or abroad, or from public or private research centers.
L'archive ouverte pluridisciplinaire HAL, est destinée au dépôt et à la diffusion de documents scientifiques de niveau recherche, publiés ou non, émanant des établissements d'enseignement et de recherche français ou étrangers, des laboratoires publics ou privés. 


\title{
Direct numerical simulation of a reacting turbulent channel flow with thermo-chemical ablation
}

\author{
Olivier Cabrit ${ }^{\mathrm{a} *}$ and Franck Nicoud ${ }^{\mathrm{b}}$ \\ ${ }^{a}$ CERFACS, 42 avenue Gaspard Coriolis, 31057 Toulouse, France; \\ b Université Montpellier 2, place Eugène Bataillon, 34095 Montpellier, France
}

(accepted version)

\begin{abstract}
This study presents the results obtained by performing a set of direct numerical simulations (DNS) of periodic channel flows over an isothermal surface subjected to thermo-chemical ablation. A specific boundary condition to handle the surface ablation is developed and allows to represent any kind of ablation scheme. By nature, the periodic channel flow configuration is statically unsteady when ablation is present, which requires a particular attention when investigating flow statistics. An ensemble average over several flow realizations is then employed to generate converged statistics. Hence, this procedure allows to investigate different features of the flow when focussing on species/momentum/energy/atomic conservation. The analysis reveals that the flow can be considered at chemical equilibrium under the conditions investigated. Moreover, the convective effect introduced by the Stephan velocity at ablative surface appears to have no influence on momentum conservation whereas it strongly participates to the surface cooling effect. Finally, an atomic mass conservation equation of Shvab-Zel'dovich type is formulated, and highlights passive scalar like conservation mechanism of atomic elements.
\end{abstract}

Keywords: Turbulent boundary layer, Reacting compressible flow, Ablation

\section{Introduction}

Ablative surface flows often arise when using thermal protection materials for preserving structural components of atmospheric re-entry spacecrafts[1, 2] and Solid Rocket Motors (SRM) internal insulation or nozzle assembly[3-5]. Depending on the structure material, ablation can occur under the combination of different physical actions: 1) oxidation of the material surface by flow traveling gaseous species; 2) pyrolysis of the composite material resin (series of chemical reactions arising in the material itself); 3) sublimation of the material at high temperature; 4) mechanical erosion due to pressure or shear stress and to the impingement of solid/liquid particles on the material (for instance metal oxide particles such as $\mathrm{Al}_{2} \mathrm{O}_{3(l)}$ ). The description of surface ablation is consequently very complex to model and requires knowledges and expertise in several disciplines such as chemistry and multicomponent physics, multi-phase flow dynamics, thermo-structural mechanics of composite materials, physics of particle/droplet impingement, roughness interaction mechanisms, or physics of radiative heat transfer. For this reason, ablation process has been studied for more than forty years[6] following numerous angles of view. Despite huge scientific research efforts, a unique model able to describe the whole

\footnotetext{
*Corresponding author. Email: olivier.cabrit@cerfacs.fr
} 
phenomenon does not exist. It is thus essential to properly define the assumptions and the framework of each investigation.

The present work is mainly motivated by the need to improve the understanding of momentum and heat transfer towards SRM nozzle structure. In this application, the use of high energy solid propellant generates an hostile environment and the nozzle structure is then exposed to severe thermochemical attack. Graphite and carbon-carbon composites are widely used because they offer excellent thermomechanical properties for low material density. By the nature of these materials, classified as non-charring materials, pyrolysis does not occurs and ablation is mainly due to the oxidizing species of the combustion products such as $\mathrm{H}_{2} \mathrm{O}, \mathrm{CO}_{2}, \mathrm{H}_{2}$ or $O H$. As a consequence, the heterogeneous surface reactions make the nozzle surface to recede which is an issue during motor firing since the SRM performance is lowered by the throat area increase and the apparition of surface roughness[7]. However, this solid material sacrifice can also be seen has a desirable effect since the wall normal convection induced at the nozzle surface counters the energy flux that would have otherwise entered the nozzle structure. Hence, it is crucial to control the surface ablation during nozzle design to establish a good compromise between nozzle recession and structure cooling.

Full-scale motor firings are very expensive and do not provide sufficient information to understand the whole phenomenon. Moreover, because of technological difficulties (high temperature and pressure), the classical experimental techniques for surface ablation study[4] do not give reliable data concerning the nozzle boundary layer structure. For these reasons, numerical simulations are widely used in the field of ablation investigation. Many studies have already proposed to couple numerically the gaseous phase and the solid structure[5, 8-13]. However, most of them are dedicated to the structural material characterization by predicting the recession rate or the surface temperature and few are oriented toward the fluid characterization. Moreover, these studies make use of Reynolds averaged Navier-Stokes (RANS) methods for which the near wall turbulence structures are intrinsically presumed. Hence, the objective of the present study is to support the understanding of turbulent boundary layer structure over ablative surfaces by the use of direct numerical simulation (DNS).

The classical periodic channel flow configuration[14] has widely demonstrated its capacity to accurately reproduce the wall turbulence structures $[15,16]$ and generate precise and detailed data set of generic turbulent flows under realistic operating conditions. For this reason, many authors have used this configuration to study the statistics of different types of flows such as classical incompressible flows[17], supersonic flows[18], fluids with variable properties[19] and heat transfer[20, 21], multicomponent reacting flows[22] and transpiring surface flows[23, 24]. In the present study, the compressible multicomponent reacting turbulent configuration of Cabrit and Nicoud[22] is used has a reference case for flow over inert walls. For ablative wall simulations, the no-slip wall boundary condition is merely changed to an isothermal ablative surface condition. Contrary to the approach of Velghe et al.[25], this work is performed under the assumption that surface roughness is not generated by ablation during the simulation. Sublimation of the structure material is not discussed in the present study since this phenomenon usually appears for temperatures over $4000 \mathrm{~K}$, which corresponds to an higher regime than the one considered herein. Moreover, the question of two-phase flow effects and mechanical erosion is not addressed even if recent studies have shown the influence of these phenomena[26, 27]. This assumption is supported by the experimental study of Klager[28] which has demonstrated that chemical attack is the primary source of erosion. To summarize, the present study only focusses on ablation by chemical 
oxidation of the surface. It is shown how this surface characteristic influences the momentum/energy/atomic conservation by analyzing the corresponding balances for three operating conditions of the ablation boundary condition. The influence of both the oxidation scheme and the wall normal Stephan velocity is investigated.

The paper is arranged as follows. Section 2 presents the theoretical and numerical background. Particular attention is given to the ablation boundary condition description which has been developed for this study. Section 3 explains and justifies the ensemble average statistical procedure retained for this study. Section 4 provides the analysis of the boundary layer structure. Finally, the conclusions of this work are drawn in section 5 .

\section{Description of the simulations}

\subsection{Flow equations}

\subsubsection{Conservation laws}

Several books[29-31] present the conservation laws that model the physics of multicomponent reacting compressible flows. In the present study one makes use of the same formalism and notation as in reference [22]. Continuity equation (1), mass species conservation (2), momentum conservation (3), total non-chemical energy conservation (4) and perfect gas equation of state (5) then reads (with Einstein notation for implicit summation):

$$
\begin{gathered}
\frac{\partial \rho}{\partial t}+\frac{\partial\left(\rho u_{i}\right)}{\partial x_{i}}=0 \\
\frac{\partial\left(\rho Y_{k}\right)}{\partial t}+\frac{\partial}{\partial x_{i}}\left(\rho\left(u_{i}+V_{k, i}\right) Y_{k}\right)=\dot{\omega}_{k} \\
\frac{\partial\left(\rho u_{i}\right)}{\partial t}+\frac{\partial\left(\rho u_{i} u_{j}\right)}{\partial x_{j}}=-\frac{\partial p}{\partial x_{i}}+\frac{\partial \tau_{i j}}{\partial x_{j}}+\mathcal{S}_{i} \\
\frac{\partial \rho E}{\partial t}+\frac{\partial \rho u_{i} E}{\partial x_{i}}=-\frac{\partial q_{i}^{*}}{\partial x_{i}}+\frac{\partial}{\partial x_{j}}\left(\tau_{i j} u_{i}\right)-\frac{\partial}{\partial x_{i}}\left(p u_{i}\right)+\dot{\omega}_{T}+\mathcal{Q}+u_{i} \mathcal{S}_{i} \\
\frac{p}{\rho}=r T
\end{gathered}
$$

with $\tau_{i j}$ the viscous shear stress tensor and $q_{i}$ the molecular heat flux (different from $q_{i}^{*}$ that represents the sensible enthalpy flux) respectively given by:

$$
\tau_{i j}=-\frac{2}{3} \mu \frac{\partial u_{l}}{\partial x_{l}} \delta_{i j}+\mu\left(\frac{\partial u_{i}}{\partial x_{j}}+\frac{\partial u_{j}}{\partial x_{i}}\right)
$$




$$
q_{i}=\underbrace{-\lambda \frac{\partial T}{\partial x_{i}}+\rho \sum_{k} h_{s, k} Y_{k} V_{k, i}}_{q_{i}^{*}}+\rho \sum_{k} \Delta h_{f, k}^{0} Y_{k} V_{k, i}
$$

The following notation stands for the present study: $i$ subscripted quantities refer to variables dependent on directions $\vec{x}, \vec{y}$ and $\vec{z}$ (for multiple summation, subscript $i$ is replaced by either $j$ or $l$ ); subscript $k$ refers to $k^{t h}$ species; $u_{i}$ is the velocity vector whose components are noted $u, v$ and $w$ in the cartesian coordinate system; $p$ is the thermodynamic pressure; $T$ the temperature; $\rho$ the density of the fluid; $Y_{k}$ and $X_{k}$ denotes the mass and molar fractions of species $k$ respectively, with $Y_{k}=X_{k} W_{k} / W ; W_{k}$ the atomic weight of species $k ; W=\sum_{k} X_{k} W_{k}$ the mean molecular weight of the mixture; $r=R / W$ the perfect gas constant per mass unit with $R$ the perfect gas constant per mole; $V_{k, i}$ the diffusion velocity of species $k$ in $i$-direction; $\dot{\omega}_{k}$ the mass reaction rate of species $k ; \dot{\omega}_{T}=-\sum_{k} \Delta h_{f, k}^{0} \dot{\omega}_{k}$ is the heat release with $\Delta h_{f, k}^{0}$ the chemical enthalpy of formation per unit mass of species $k ; \mathcal{S}_{i}$ the momentum source term acting in the $i$-direction and $\mathcal{Q}$ the energy volume source term; $C_{p, k}$ the heat capacity at constant pressure of species $k ; C_{p}=\sum_{k} C_{p, k} Y_{k}$ and $C_{v}$ the heat capacities at constant pressure and constant volume of the mixture, respectively; $\mu$ and $\nu=\mu / \rho$ are the dynamic and the kinematic viscosities, respectively; $\lambda$ the heat diffusion coefficient of the fluid; $E$ is the total non-chemical energy; $h_{s, k}=\int_{T_{0}}^{T} C_{p, k} d T$ represents the sensible enthalpy of one species $k$ with a reference temperature $T_{0}=0 K$ in this work; $h_{k}=h_{s, k}+\Delta h_{f, k}^{0}$ is the specific enthalpy (sum of sensible and chemical parts) of one species $k$; the enthalpy of the mixture is represented by the specific enthalpy $h=h_{s}+\sum_{k} \Delta h_{f, k}^{0} Y_{k}$ with $h_{s}=\sum_{k} h_{s, k} Y_{k}$ the sensible enthalpy of the mixture.

This system of equations (1-7) is valid under the following assumptions:

- buoyancy body force is negligible,

- effects of volume viscosity are null,

- no Dufour effect for the heat flux,

- the gas mixture is supposed to be perfect,

- heat transfer by radiation is negligible.

The validity of these assumptions has been discussed and justified in a previous study[22] that simulated the same gaseous flow under the same thermodynamic conditions over inert walls. Moreover, concerning the validity of neglecting the radiative heat transfer, Amaya et al.[32] demonstrated that the radiative source term has no influence upon the turbulence characteristics of the flow.

\subsubsection{Multicomponent transport modeling}

When diffusion due to external forces, pressure and temperature gradients are neglected, the expression of the diffusion velocity is reduced to:

$$
V_{k, i}=-\sum_{l} D_{k l} \frac{\partial X_{l}}{\partial x_{i}}
$$

where $D_{k l}$ are the multicomponent diffusion coefficients of the diffusion matrix. Note that the Soret effect (diffusion due to temperature gradients) is well negligible according to the a priori tests performed in reference[22]. Solving this transport system Eq. (8) could be very expensive when the number of considered species is 
high. For this reason, the transport system is often simplified making use of the Hirschfelder and Curtiss approximation[33] with correction velocity. As mentioned by Giovangigli[34] this is the best first-order acurate model for estimating diffusion velocities of a multicomponent mixture. The diffusion velocity system is then replaced by:

$$
V_{k, i}^{\mathrm{hc}} X_{k}=-\mathcal{D}_{k} \frac{\partial X_{k}}{\partial x_{i}}
$$

where $V_{k, i}^{\text {hc }}$ denotes the Hirschfelder and Curtiss diffusion velocity, and $\mathcal{D}_{k}$ an equivalent diffusion coefficient of species $k$ into the rest of the mixture. The latter coefficient is built from the binary diffusion coefficients $\mathcal{D}_{i j}^{\text {bin }}$ which can be assessed from the gas kinetic theory[34]:

$$
\mathcal{D}_{k}=\frac{1-Y_{k}}{\sum_{j \neq k} X_{j} / \mathcal{D}_{j k}^{\mathrm{bin}}}
$$

Moreover, to insure that the system of equations satisfies the constraint $\sum_{k} Y_{k} V_{k, i}=0$, a correction velocity $V_{i}^{\text {cor }}$ is added to the Hirschfelder and Curtiss diffusion velocity $V_{k, i}^{\mathrm{hc}}$. At each time step, the correction velocity is computed as:

$$
V_{i}^{\text {cor }}=\sum_{k} \mathcal{D}_{k} \frac{W_{k}}{W} \frac{\partial X_{k}}{\partial x_{i}}
$$

so that the diffusion velocity for each species $k$ reads:

$$
V_{k, i}=V_{k, i}^{\mathrm{hc}}+V_{i}^{\mathrm{cor}}
$$

Combined with the assumption of constant Schmidt numbers, the Hirschfelder and Curtiss approximation is very convenient because the equivalent diffusion coefficients can be easily related to the kinematic viscosity according to: $\mathcal{D}_{k}=\nu / S c_{k}$. The problem is then efficiently closed by imposing the Schmidt numbers and it is not necessary to compute the $\mathcal{D}_{i j}^{\text {bin }}$ coefficients which are complex functions of collision integrals and thermodynamics variables.

The mixture retained in this study has been built by simplifying a realistic sample of gas ejected from a SRM nozzle. About a hundred gaseous species usually compose this kind of mixture. However, only the ones whose mole fraction is greater than 0.001 have been kept to generate a simpler mixture, nitrogen being used as diluent. Hence, the following seven species are retained for the simulation: $\mathrm{H}_{2}, \mathrm{H}, \mathrm{H}_{2} \mathrm{O}$, $\mathrm{OH}, \mathrm{CO}_{2}, \mathrm{CO}$ and $\mathrm{N}_{2}$. No aluminized particles are thus considered even if their importance on graphite nozzle erosion is a current active topic in the literature. Indeed, the experiments of Klager[28], Geisler[3] and Cvelbar[35] with aluminized composite solid propellant, as well as the numerical analysis of Keswani et al.[36] have indicated that the recession rate decreases when aluminum concentration increases. However, these studies also reveal that this phenomenon was strongly correlated with the associated decreased concentration of oxidizing species $\mathrm{H}_{2} \mathrm{O}$ and $\mathrm{CO}_{2}$. This means that aluminized species rather have an indirect impact on surface ablation which is not addressed in the current study.

The Schmidt and Prandtl numbers are determined using the EGLIB library [37, 38] and summarized in Table 1. They are assumed to be constant in the simulation which is a well accepted assumption[31]. The same multicomponent library has 
Table 1. Transport coefficients of the simulated mixture.

\begin{tabular}{cccccccc}
\hline $\operatorname{Pr}$ & $S c_{\mathrm{H}_{2}}$ & $S c_{\mathrm{H}}$ & $S c_{\mathrm{H}_{2} \mathrm{O}}$ & $S c_{\mathrm{OH}}$ & $S c_{C O_{2}}$ & $S c_{C O}$ & $S c_{N_{2}}$ \\
\hline 0.47 & 0.2 & 0.15 & 0.65 & 0.54 & 0.98 & 0.86 & 0.87 \\
\hline
\end{tabular}

Table 2. Chemical kinetic scheme retained for this study. The first and fifth reactions involve an abstract third body, denoted by $M$. For these reactions the third-body efficiencies are set by default to $\alpha_{k, r}=1.0$ for all the species, except for the ones given in the table.

\begin{tabular}{|c|c|c|c|c|c|c|}
\hline \multicolumn{3}{|c|}{ Reaction } & $A[$ cgs units $]$ & $\beta$ & $E_{a}[\mathrm{cal} / \mathrm{mole}]$ & $\alpha_{k, r}$ \\
\hline $2 H+M$ & $\rightleftharpoons$ & $H_{2}+M$ & $1.00 \mathrm{E}+18$ & -1.0 & 0. & $\alpha_{H_{2}}=\alpha_{H_{2} O}=0.0$ \\
\hline $2 \mathrm{H}+\mathrm{H}_{2}$ & $\rightleftharpoons$ & $2 \mathrm{H}_{2}$ & $9.00 \mathrm{E}+16$ & -0.6 & 0. & - \\
\hline $2 \mathrm{H}+\mathrm{H}_{2} \mathrm{O}$ & $\rightleftharpoons$ & $\mathrm{H}_{2}+\mathrm{H}_{2} \mathrm{O}$ & $6.00 \mathrm{E}+19$ & -1.25 & 0. & - \\
\hline $2 \mathrm{H}+\mathrm{CO}_{2}$ & $\rightleftharpoons$ & $\mathrm{H}_{2}+\mathrm{CO}_{2}$ & $5.50 \mathrm{E}+20$ & -2.0 & 0. & - \\
\hline$H+O H+M$ & $\rightleftharpoons$ & $\mathrm{H}_{2} \mathrm{O}+\mathrm{M}$ & $2.20 \mathrm{E}+22$ & -2.0 & 0. & $\alpha_{H_{2}}=0.73 ; \alpha_{H_{2} O}=3.65$ \\
\hline $\mathrm{OH}+\mathrm{H}_{2}$ & $\rightleftharpoons$ & $\mathrm{H}+\mathrm{H}_{2} \mathrm{O}$ & $2.16 \mathrm{E}+08$ & 1.51 & 3430. & $-1+20$ \\
\hline $\mathrm{OH}+\mathrm{CO}$ & $\rightleftharpoons$ & $\mathrm{H}+\mathrm{CO}_{2}$ & $4.76 \mathrm{E}+07$ & 1.228 & 70. & - \\
\hline
\end{tabular}

been used to compute the temperature dependancy of the mixture dynamic viscosity. A power-law expression is finally retained:

$$
\mu=\mu_{\mathrm{ref}}\left(\frac{T}{T_{\mathrm{ref}}}\right)^{c}
$$

where $\mu_{\text {ref }}=8.194 \times 10^{-5}$ Pa.s, $T_{\text {ref }}=3000 K$ and $c=0.656$.

\subsubsection{Chemical kinetics of the mixture}

The simulated mixture needs a reliable kinetic scheme which reproduces the concentration changes of each species during the simulations. Hence, a kinetic scheme based on seven chemical reactions has been tuned using the GRI-Mech elementary equations (URL: http://www.me.berkeley.edu/gri_mech). Details of the retained scheme are presented in Table 2 where the rate of reaction, $K$, is modeled by a classical Arrhenius formulation[34]: $K=A T^{\beta} \exp \left(-E_{a} / R T\right)$, where $A$ stands for the pre-exponential factor, $\beta$ for the temperature exponent and $E_{a}$ for the activation energy. This kinetic scheme has been validated verifying its ability to predict the proper chemical equilibrium composition as given by the EQUIL library of CHEMkin $[39,40]$ and the whole set of species involved in GrI-Mech chemical reaction mechanism. The same kinetic scheme was also used in [22].

\subsection{Boundary condition for surface ablation}

The development of the boundary condition for surface ablation is performed under the following assumptions: no mechanical erosion, no geometry deformation. Note also that by the nature of $C / C$ composite materials, it is not necessary to take into account any pyrolysis modeling. Hence, one supposes that the near wall flow description will be sufficiently and accurately predicted by considering the effect of heterogeneous oxidation reactions only.

The ablation process is simplified following the assumption used by Baiocco and Bellomi[11], namely the injection velocity (also called Stephan velocity) is assumed to be orthogonal to the receding surface (tangential components are set to zero). Hence, this new boundary condition prescribes the boundary values for the conservation equations (of mass, momentum, species and energy) to mimic the behavior of an ablated isothermal wall. The three following quantities are thus imposed: $v_{i n j}$ the wall normal Stephan velocity, $\partial Y_{k, w} / \partial n$ the wall normal mass 
fraction flux of each species involved in the heterogeneous reactions, and $T_{w}$ the temperature at the wall.

The boundary condition is inspired by the wall recession model proposed by Keswani and Kuo[9, 36] and the work of Kendall et al. [8]. The analytical development that follows is done in the relative referential of the surface and not in the global coordinate system. This means that all the equations presented herein are projected on the wall normal directed toward the gaseous domain by convention. Starting from the conservation equation of the mass flux, $\dot{m}$, at the fluid/structure interface, one can write:

$$
\dot{m}=\rho_{c} \dot{r}_{c}=\rho_{w} v_{i n j}
$$

where $\rho_{c}$ denotes the mass density of the composite material, $\dot{r}_{c}$ the recession rate of the solid surface, and $\rho_{w}$ the gas density at the wall. Equation (14) indicates that the recession solid velocity is linked to the Stephan velocity through the ratio of solid and gas densities: $v_{i n j} / \dot{r}_{c}=\rho_{c} / \rho_{w}$. Considering the carbon density to be about $\rho_{c} \approx 1900 \mathrm{~kg} \cdot \mathrm{m}^{-3}$ and the gas density to be of order $\rho_{w} \approx 7 \mathrm{~kg} \cdot \mathrm{m}^{-3}$, one finds that the solid surface moves several hundreds times slower than the heterogeneous reaction gas entering inside the gaseous domain. This justifies not to take into account the structure deformation when investigating the gaseous phase behavior. Focussing on the species conservation balance at the fluid/structure interface one can write the same kind of balance:

$$
\rho_{w} v_{i n j} Y_{k, w}+\rho_{w} V_{k, n, w} Y_{k, w}=\dot{s}_{k}
$$

where $Y_{k, w}$ stands for the mass fraction of species $k$ at the wall, $V_{k, n, w}$ the wall normal diffusion velocity of species $k$ at the wall, and $\dot{s}_{k}$ the surface mass production rate of species $k$ that depends on the chemical heterogeneous reactions involved in the oxidation process (the modeling of $\dot{s}_{k}$ is presented further in this section). Summing over all the species, and according to the mass conservation constraint $\sum_{k} Y_{k} V_{k, i}=0$, an expression for the wall normal Stephan velocity is obtained:

$$
v_{i n j}=\frac{1}{\rho_{w}} \sum_{k} \dot{s}_{k}
$$

The Hirschfleder and Curtiss approximation with correction velocity, equations $(9,11,12)$, are then used to evaluate the normal diffusion flux of each species at the ablated wall:

$$
Y_{k, w} V_{k, n, w}=-\mathcal{D}_{k} \frac{W_{k}}{W_{w}} \frac{\partial X_{k, w}}{\partial n}+Y_{k, w} V_{n}^{\text {cor }}
$$

with,

$$
V_{n}^{\text {cor }}=\sum_{k} \mathcal{D}_{k} \frac{W_{k}}{W_{w}} \frac{\partial X_{k, w}}{\partial n}
$$

where $W_{w}$ is the mean molecular weight of the mixture at the wall. Moreover, one knows that the relation between the mole fraction gradients and their mass fraction 
counterparts reads:

$$
\frac{\partial X_{k}}{\partial x_{i}}=\frac{W}{W_{k}} \frac{\partial Y_{k}}{\partial x_{i}}-\frac{W^{2}}{W_{k}} Y_{k} \sum_{l} \frac{1}{W_{l}} \frac{\partial Y_{l}}{\partial x_{i}}
$$

Making use of Eq. (19) and injecting equations (16), (17), and (18) into Eq. (15), one can relate $Y_{k, w}$ to its normal gradient at the boundary surface:

$$
\frac{\partial Y_{k, w}}{\partial n}=\frac{Y_{k, w}}{\mathcal{D}_{k}}\left(\frac{\sum_{l} \dot{s}_{l}}{\rho_{w}}+V_{n}^{\mathrm{cor}}+W_{w} \mathcal{D}_{k} \sum_{l} \frac{1}{W_{l}} \frac{\partial Y_{l, w}}{\partial n}\right)-\frac{\dot{s}_{k}}{\rho_{w} \mathcal{D}_{k}}
$$

where the term $V_{n}^{\text {cor }}$ has not been fully expressed as a function of $Y_{k, w}$ for clarity. Thereby, Eq. (20) constitutes a system of equations of size $n s \times n s$, ns being the number of species present in the mixture. In the present study, this system is solved thanks to an iterative method which gives for each time step the wall normal value of the mass fraction gradient that the numerical solver must satisfy. Finally, the surface temperature is imposed with a Dirichlet method.

The framework described above makes no restriction concerning the number and the nature of the chemical species or the kind of heterogeneous chemical reactions. This means that sublimation mechanisms can be implemented as well as oxidation reactions. The boundary condition for ablated walls, Eq. (20), can be summarized through the following characteristics:

- arbitrary mixture compositions and heterogeneous reactions can be considered;

- the momentum condition for $v_{i n j}$ is imposed with a Dirichlet method according to Eq. (16), tangential components being set to zero;

- the variations of species concentrations are accounted for with the Neumann condition of Eq. (20);

- the surface temperature, $T_{w}$, is imposed by a Dirichlet condition.

The final closure of the boundary condition is insured by the model retained for the surface production rates, $\dot{s}_{k}$, which depends on the heterogeneous reaction considered. One first recalls that sublimation is not considered in the present study because the temperature of investigation is too low (temperature higher than $4000 \mathrm{~K}$ is required[41]). Experimental studies[28, 42] have however shown that chemical attack arises by species such as $\mathrm{H}_{2} \mathrm{O}, \mathrm{CO}_{2}, \mathrm{OH}, \mathrm{H}_{2}, \mathrm{O}_{2}$ and $\mathrm{O}$ for the pressure and temperature considered herein (see section 2.3). The oxidation by species $\mathrm{O}_{2}$ and $O$ is often negligible because of the weak concentration of these species. Besides, note that they do not appear in the equivalent mixture formulated above. Moreover, as reported by Thakre and Yang[5], there is a disagreement between many investigators concerning the importance of the oxidation reaction by $\mathrm{H}_{2}$ which justifies not to consider this reaction. Finally, we also neglect the oxidation of solid carbon by $O H$ species because Chelliah et al.[43] reported two orders of magnitude between the pre-exponential factor of this oxidation reaction and the ones of the oxidation reactions by $\mathrm{H}_{2} \mathrm{O}$ and $\mathrm{CO}_{2}$.

For these reasons, one only considers the two predominant oxidation reactions of $C / C$ composite in SRM nozzle flow, namely:

$$
\mathrm{C}_{(s)}+\mathrm{H}_{2} \mathrm{O} \rightarrow \mathrm{H}_{2}+\mathrm{CO}
$$




$$
\mathrm{C}_{(s)}+\mathrm{CO}_{2} \rightarrow 2 \mathrm{CO}
$$

These two reactions have been extensively studied[43-46], and one usually makes use of a classical Arrhenius law for modeling their rate of reaction. The resulting model for the progress of the first reaction (oxidation by $\mathrm{H}_{2} \mathrm{O}$ ) can be written as:

$$
\mathcal{Q}_{1}=\rho_{w} \frac{Y_{H_{2} O, w}}{W_{H_{2} O}} A_{1} T_{w}^{\beta_{1}} \exp \left(\frac{-E_{a, 1}}{R T_{w}}\right)
$$

and for the second reaction (oxidation by $\mathrm{CO}_{2}$ ):

$$
\mathcal{Q}_{2}=\rho_{w} \frac{Y_{C O_{2}, w}}{W_{C O_{2}}} A_{2} T_{w}^{\beta_{2}} \exp \left(\frac{-E_{a, 2}}{R T_{w}}\right)
$$

However, the empirical determination of the Arrhenius coefficients does not always provide sufficiently reliable results. For instance, the pre-exponential factor suggested by Golovina[44] is about an order of magnitude less than the one of Libby and Blake[45]. One thus decides not to impose the values of the Arrhenius coefficients as it is usually done[5], but rather to make use of the fact that the surface temperature is assumed constant during the whole simulation. This means that the Arrhenius parts of equations (23) and (24) is no longer dependent on the surface temperature. The rates of reaction can thus be seen as constants for the simulation and equations (23) and (24) are reduced to:

$$
\begin{aligned}
& \mathcal{Q}_{1}=\rho_{w} \frac{Y_{\mathrm{H}_{2} \mathrm{O}, w}}{W_{\mathrm{H}_{2} \mathrm{O}}} K_{1} \\
& \mathcal{Q}_{2}=\rho_{w} \frac{Y_{\mathrm{CO}_{2}, w}}{W_{C O_{2}}} K_{2}
\end{aligned}
$$

where $K_{1}$ and $K_{2}$ are the two constants that close the problem and that must be specified. The surface production rate of species $k$ due to $r$ reactions is expressed as:

$$
\dot{s}_{k}=W_{k} \sum_{r} \nu_{k, r} \mathcal{Q}_{r}
$$

where $\nu_{k, r}$ is the difference between the backward and forward molar stoichiometric coefficients of reaction $r$. Keswani and Kuo[47] have shown that $\mathrm{H}_{2} \mathrm{O}$ is the dominant oxidizing species and that oxidation by $\mathrm{CO}_{2}$ is the second important reaction. In order to investigate this aspect, one uses two types of oxidation scheme in this paper. The first scheme solely models the oxidation by $\mathrm{H}_{2} \mathrm{O}$. In this case, knowing the initial values of the Stephan velocity at the beginning of the computation, $v_{i n j}^{i}$, and the initial mass fraction of water at the wall, $Y_{H_{2} O, w}^{i}$, the expression for $K_{1}$ can be retrieved replacing $\dot{s}_{k}$ of Eq. (16) by Eq. (27):

$$
K_{1}^{1 \text { reac }}=\frac{v_{i n j}^{i}}{W_{C}} \frac{W_{H_{2} O}}{Y_{H_{2} O, w}^{i}}
$$

where $K_{1}^{1}$ reac is the constant value imposed all along the simulation when only the first oxidation reaction Eq. (21) is considered. 
The second oxidation scheme employed in this study involves the oxidation reactions by $\mathrm{H}_{2} \mathrm{O}$ and $\mathrm{CO}_{2}$ simultaneously. As advanced by Libby and Blake[45], the specific rate of both reactions can be considered to be equal which means that $K_{1}=K_{2}$. Knowing the initial oxidizing species concentrations at the wall and the initial Stephan velocity, and rearranging Eq. (27) into Eq. (16) one shows that both rates of reaction must satisfy the relation:

$$
K_{1}^{2 \text { reac }}=K_{2}^{2 \text { reac }}=\frac{v_{i n j}^{i}}{W_{C}\left(\frac{Y_{H_{2} O, w}^{i}}{W_{H_{2} O} \mathrm{O}}+\frac{Y_{\mathrm{CO}_{2}, w}^{i}}{W_{\mathrm{CO}_{2}}}\right)}
$$

where $K_{1}^{2 \text { reac }}$ and $K_{2}^{2}$ reac are imposed as constants all along the simulation when the two oxidation reactions, Eq. (21) and Eq. (22), are considered.

This method presents the advantage of being free of the experimental determination of the Arrhenius coefficients. Moreover, the use of equations (28) and (29) allows to control the initial value of the Stephan velocity. This is convenient since $v_{i n j}^{i}$ can then be set in agreement with available data in actual SRM.

\subsection{Operating conditions and computational domain}

The aim of this work is to study the influence of surface ablation upon the flow features under the configuration of periodic channel flow[14]. Figure 1 shows the reference coordinate system and the notation used to describe the computational domain. Periodic boundary conditions are applied in the homogeneous streamwise $(\vec{x})$ and spanwise $(\vec{z})$ directions. Table 3 presents the four cases investigated. The first one, noted $I$, is the reference case for this study because it corresponds to an inert wall simulation (case $B$ of Cabrit and Nicoud[22]). The three others cases correspond to ablative wall simulations. The comparison between cases $A 1$ and $A 2$ will give information concerning the influence of the oxidation scheme, while the comparison between cases $A 2$ and $A 3$ will inform about the influence of the injection velocity. The reference injection velocity for this work is set to $v_{i n j}^{\text {ref }}=0.0428 \mathrm{~m} \cdot \mathrm{s}^{-1}$ which is a typical value for SRM nozzle application. However, when the results are similar for all the ablative wall simulations, case $A 2$ will be preferably shown because this case contains more physics than the others because of its two-reaction oxidation scheme. Note in table 3 that the time is not an homogeneous dimension for the ablative wall simulations because the periodic conditions does not allow to balance the mass flux entering the domain when the walls are ablated. As a consequence, the mean mass of the domain continuously increases and the flow statistics are susceptible to be time dependent. This means that the statistical treatment must be performed with care as discussed in the forthcoming section 3 . At this step, one specifies that for any variable $f$, the quantity $\bar{f}$ represents its value averaged in the homogeneous directions. This notation will be explicated in detail in section 3 .

The pressure gradient that drives the flow and compensates for viscous dissipation is enforced by adding a space and time constant source term in the streamwise direction. Hence, the momentum source term $\mathcal{S}_{i}$ of Eq. (3) and Eq. (4) is null in the wall normal and spanwise directions but takes a constant value, noted $\mathcal{S}_{x}$, in the streamwise direction.

In addition, a source term, noted $\mathcal{Q}$, that warms the fluid in volume is added to the energy equation (4) in order to drive the mean temperature of the wall-bounded flow to the desired value $T_{\text {mean }}$. At each time step, a space constant value of $\mathcal{Q}$ is 


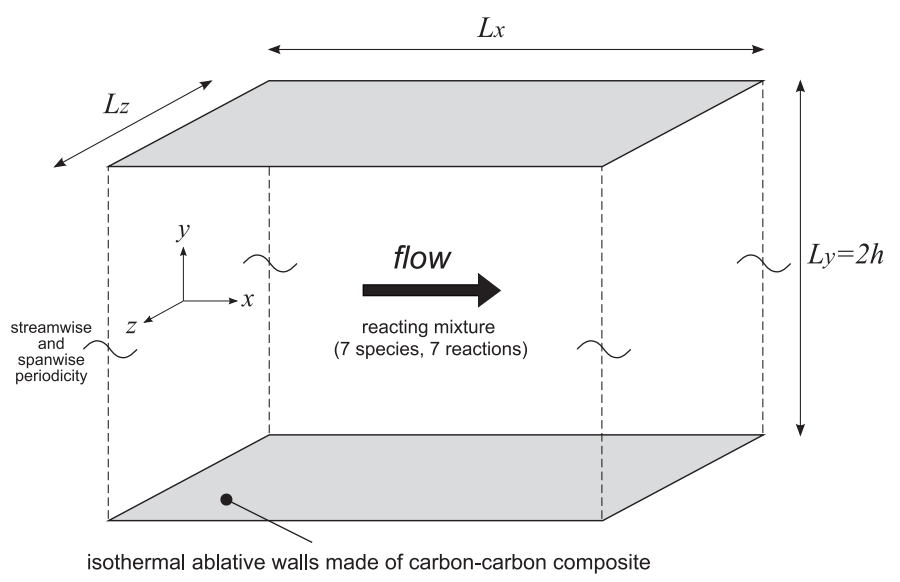

Figure 1. Description of the computational domain.

Table 3. Description of the cases of investigation.

\begin{tabular}{cccc}
\hline cases & surface oxidation scheme & initial Stephan velocity, $v_{i n j}^{i}$ & homogeneous directions \\
\hline I & none (inert wall) & none & $x, z, t$ \\
A1 & 1 reaction, Eq. (28) & $v_{i n j}^{\text {ref }}$ & $x, z$ \\
A2 & 2 reactions, Eq. (29) & $v_{i n j}^{\text {ref }}$ & $4 v_{i n j}^{\text {ref }}$ \\
A3 & 1 reaction, Eq. (28) & & $x, z$ \\
\hline
\end{tabular}

adjusted dynamically according to:

$$
\mathcal{Q}^{t+1}=\rho_{a v} C_{v, a v} \frac{T_{\text {mean }}-\frac{1}{V} \iiint_{\Omega} T^{t} d V}{\tau_{\text {relax }}}
$$

where subscript "av" denotes an averaged quantity integrated over space and time variables, $\mathcal{Q}^{t+1}$ the energy source term at time $t+\Delta t, \Omega$ the computational domain and $V$ its volume, $T^{t}$ the temperature at time $t$, and $\tau_{\text {relax }}$ a relaxation time coefficient set in the present study to $\tau_{\text {relax }}=0.3 h / \overline{u_{\tau}}$ with $\overline{u_{\tau}}=\sqrt{\overline{\tau_{w}} / \overline{\rho_{w}}}$ the mean friction velocity averaged in the homogeneous directions. The coefficient 0.3 retained for the present computations allows to maintain the target mean temperature without inducing spurious dynamical forcing on the flow statistics that could potentially occur at very low $\tau_{\text {relax }}$ values. A value taken in the range $0.2-0.5$ would have also given the same flow statistics.

For all the cases reported in table 3 , the target friction number is about $R e_{\tau}=$ $h \overline{u_{\tau}} / \nu_{w}=300$, which corresponds to a bulk Reynolds number (based on the channel half-height and bulk quantities) of $R e_{b} \approx 4200$. For all cases, the mean target temperature of the flow is set to $T_{\text {mean }}=3000 \mathrm{~K}$ and the wall surface temperature is set to $T_{w}=2750 \mathrm{~K}$. The mean pressure is around $p=10 \mathrm{MPa}$. The spatial resolution of the turbulent structures is performed on $31 \times 159 \times 39$ grid points, with $L_{x} / h=3.14$ and $L_{z} / h=1.25$. In terms of grid spacing this corresponds to $\Delta x^{+}=29$ and $\Delta z^{+}=9$, where "+" notation refer to a scaling by the viscous length $y_{\tau}=\nu_{w} / \overline{u_{\tau}}$. The mesh is refined in the vicinity of the wall in order to well capture the turbulence created in this region. The minimum and maximum grid spacing in the wall normal direction are about $\Delta y_{w}^{+}=0.8$ at the wall and $\Delta y_{c}^{+}=6$ at the center of the channel. The length of the computational box and the number of grid points seem to be very small compared to the reference simulation of Hoyas and Jiménez[17]. However, the study of Cabrit and Nicoud[22] has demonstrated that this computational domain is sufficient to capture the first order statistics of this 


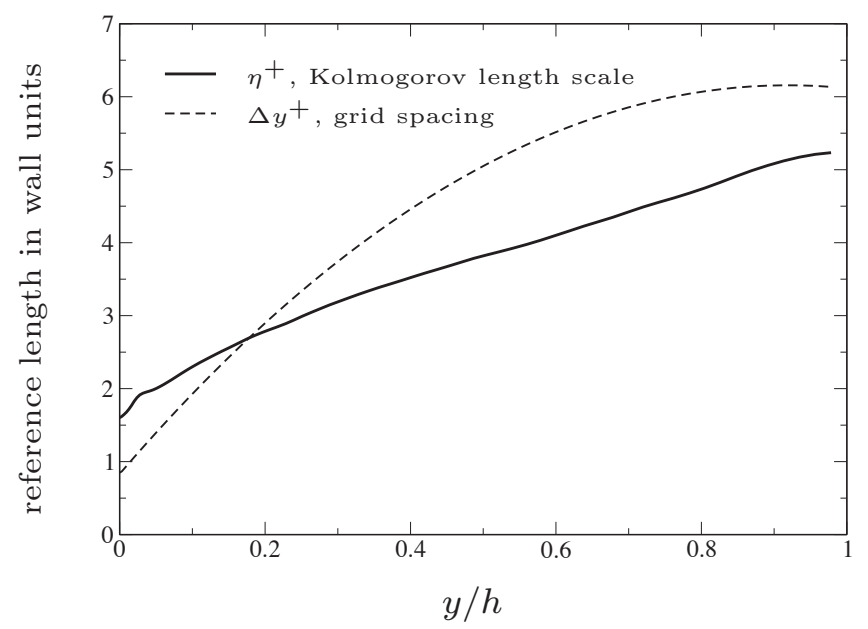

Figure 2. Wall normal grid spacing of the computational domain compared to the Kolmogorov length scale.

reacting compressible flow. Indeed, the domain has been built to be at least twice wider than the minimal unit flow conditions defined by Jiménez and Moin[48].

The numerical fidelity of the calculations has been checked looking at the wall normal evolution of the Kolmogorov length scale compared to the grid spacing. This diagnostic is presented in figure 2 for case $A 2$, where the Kolmogorov length scale is estimated as: $\eta=\left(\bar{\nu}^{3} / \bar{\epsilon}\right)^{1 / 4}$, with $\bar{\epsilon}$ the mean dissipation rate of the turbulent kinetic energy. This figure shows that the spatial resolution is very good near the wall and of the order of the Kolmogorov length scale near the center of the channel which argues in favor of an accurate flow resolution. Furthermore, the streamwise and spanwise two-point correlations from the channel centerline and near the walls are presented in figure 3 for case $A 2$. The correlation coefficients quickly approach zero indicating a sufficient spatial decorrelation to allow the use of periodic boundary conditions. Indeed, even if the correlation do not perfectly drop to zero like in reference highly-resolved periodic channel DNS's[17, 49], other studies[22, 48] have shown that the use of such moderately large boxes was sufficient to recover the low-order statistics investigated in the present study. Note that the statements presented in figures 2 and 3 for case $A 2$ are similar for all the present simulations.

\subsection{Numerical method}

DNS are performed with the AVBP solver developed at CERFACS. This parallel code offers the capability to handle unstructured or structured grids in order to solve the full 3D compressible reacting Navier-Stokes equations with a cell-vertex formulation. During the past years, its efficiency and accuracy have been widely demonstrated in both LES and DNS for different flow configurations[22, 50-52].

A set of Taylor-Galerkin schemes are available in the AVBP code to solve the flow equations described above. Notably a third order accurate formulation in both time and space is available[53] as well as a second order accurate cell-vertex implementation of the Lax-Wendroff scheme. The latter has been used to save CPU time and because the grid resolution is judged fine enough to make acceptable a second order approximation, based on the following arguments: (a) the wall normal grid spacing is similar to existing reference studies[49] $\left(0.8<\Delta y^{+}<6\right)$; (b) only low-order statistics are investigated; (c) tests conducted on the inert wall case show negligible differences between this second-order scheme and the third-order numerical scheme used in a previous study[22] (comparison not shown). 

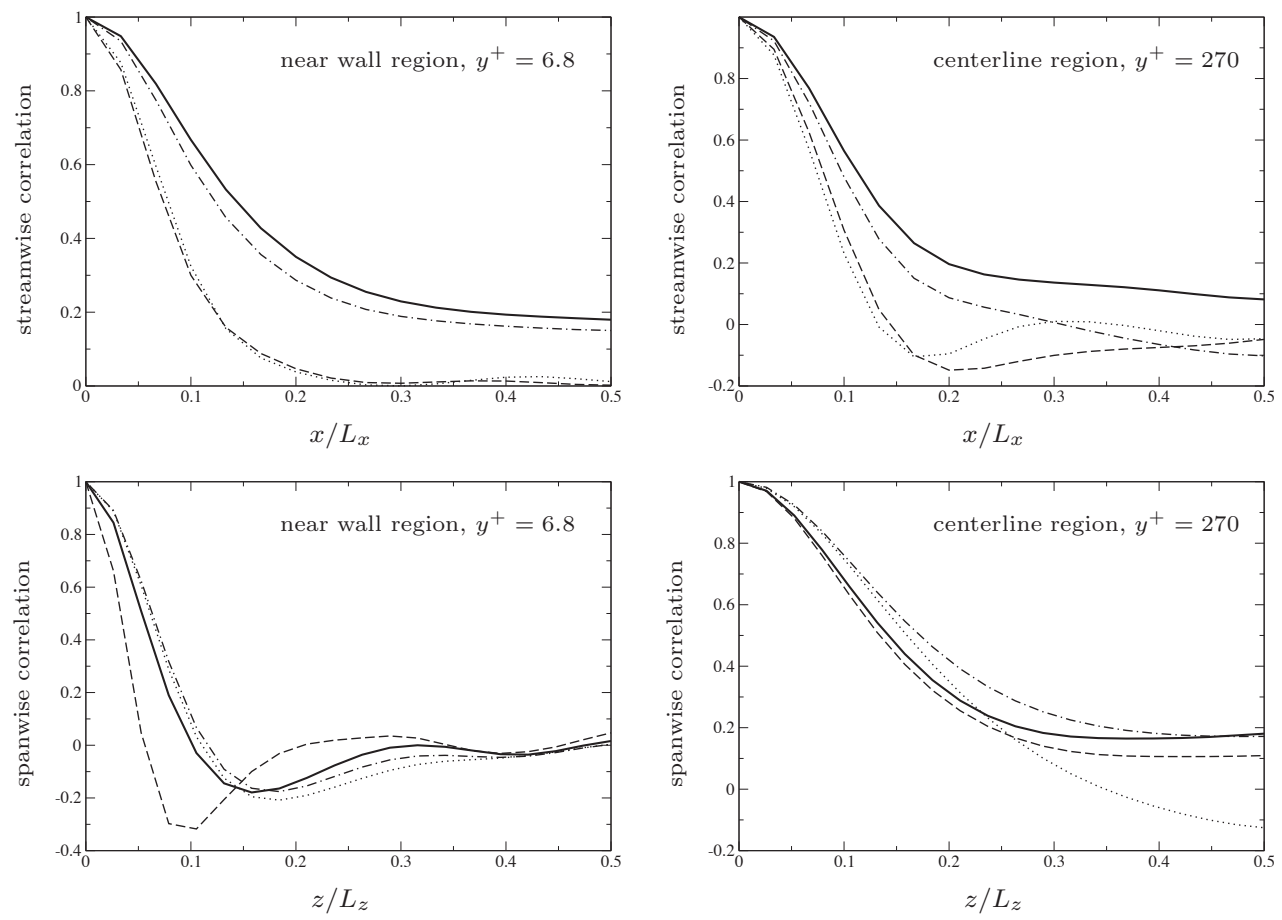

Figure 3. Two-point correlations in streamwise and spanwise directions for case A2. - $----\mathrm{v} ; \quad$....... : w; - - - : T.

\section{Statistical Procedure}

The statistics for the DNS with inert walls (case $I$ ) are collected with the classical procedure: averaging over $\vec{x}$ and $\vec{z}$ directions, and over an assimilation time $\tau_{s} \approx 20 \tau_{d}$, with $\tau_{d}=h / \overline{u_{\tau}}$. On the contrary, the DNS's with ablative walls (cases $A 1, A 2$ and $A 3)$ are statistically unsteady because the oxidation reaction at the wall consumes the $\mathrm{H}_{2} \mathrm{O}$ species initially present in the computational domain (as well as $\mathrm{CO}_{2}$ species when the second oxidation reaction is activated). Thus, the data cannot be averaged over time which, given the moderate size of the computational domain, makes the statistical convergence more challenging. For instance, the computational box size should be about a hundred times wider to obtain converged statistics at a given time. Moreover, the time advancement of the DNS is dependent on the initial condition that determines the spatial organization of the oxidizing species. For these reasons, we have performed an ensemble average from twenty different DNS's with ablative walls, differing because of the initial conditions. To insure sufficient decorrelation between the initial solutions, they are chosen from the inert wall simulation with a separating time equal to the diffusion time, $\tau_{d}$. The validity of the initial solution sample has been tested verifying that the statistics obtained with these twenty instantaneous solutions give accurate reproduction of the statistics accumulated with the full inert wall DNS (case $I$, with more than 500 instantaneous solutions). This result is presented in figure 4 for the root-mean-square (rms) velocity fluctuations.

The ensemble average procedure for the ablative wall simulations is illustrated in figure 5 where the time evolution of the injection velocity and the density at the wall is presented for one probe located on the ablated surface of case $A 2$. One observes the convergence of the average performed over twenty realizations compared to the single signal of each realization. Moreover, in order to increase the statistical sample at a given observation time, one also collects two instantaneous solution fields before and after the observation time both separated by approximatively one 


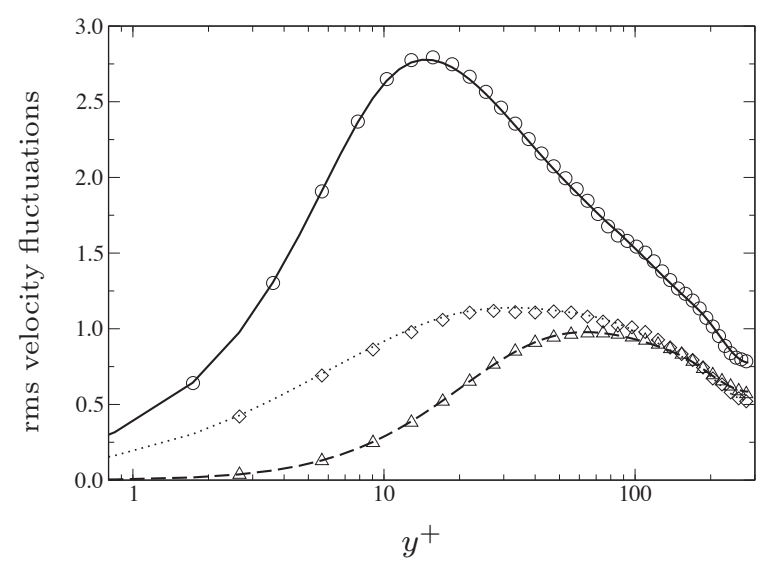

Figure 4. Root-mean-square velocity fluctuations scaled by $\overline{u_{\tau}}$. Comparison between the statistics obtained with the whole case $I$ dataset (lines) and with the twenty decorrelated solution files used to initialize the ablated wall simulations (symbols). $(-\quad, \mathrm{O}): u_{\mathrm{rms}}^{\prime} ; \quad(----, \triangle): v_{\mathrm{rms}}^{\prime} ; \quad(\cdots \cdots, \diamond): w_{\mathrm{rms}}^{\prime}$.
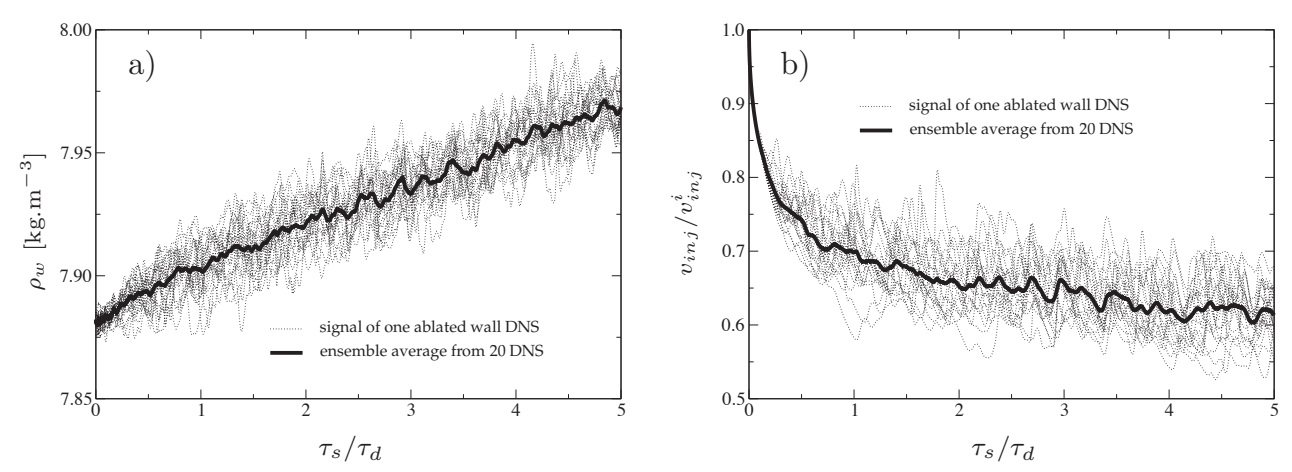

Figure 5. Evolution of the density (a) and of the injection velocity (b) for one probe located at the wall during the ablative wall simulation of case $A 2$. The velocity is scaled by the initial injection velocity, $v_{i n j}^{i}$, and the simulated time, $\tau_{s}$, is scaled by $\tau_{d}$.

Kolmogorov time scale. This means that at a given observation time, one actually makes use of sixty solutions fields to performed the ensemble average. This procedure is illustrated in figure 6 and justified in figure 7 where the separation time between the solutions used to complete the statistical sample is compared to the Kolmogorov time scale, $\tau_{\eta}=(\bar{\nu} / \bar{\epsilon})^{1 / 2}$, inside the boundary layer. This procedure is corroborated by the fact that $\tau_{\eta}<<\tau_{d}$ (see figure 7 ), and because the variations of the studied variables $\left(v_{i n j}, Y_{k}\right.$, etc...) stay negligible over $2 \tau_{\eta}$. Since these additional fields are moderately correlated, this procedure presents the advantage of filtering the higher frequencies of the statistics without perturbing their general trend governed by high energy containing low-frequencies. The proper way to improve the statistical convergence would be to either use a larger computational domain or to increase the number of independent DNS's, both solutions leading to an additional CPU cost.

Let us properly describe the notations used in the forthcoming sections. For the inert wall case $I, \bar{f}$ represents the average of variable $f$ in $\vec{x}$ and $\vec{z}$ directions and over time. For the ablative wall cases $(A 1, A 2$, and $A 3)$, the quantity $\bar{f}$ represents the average of variable $f$ in $\vec{x}$ and $\vec{z}$ directions and over the ensemble of the realization fields forming the statistical sample at a given time of observation. For all the cases, $\widetilde{f}$ represents the Favre average defined as $\widetilde{f}=\overline{\rho f} / \bar{\rho}$. The double prime, ", represents the turbulent fluctuations with respect to Favre averages.

The main difference between the simulated and the real ablative wall cases is that 


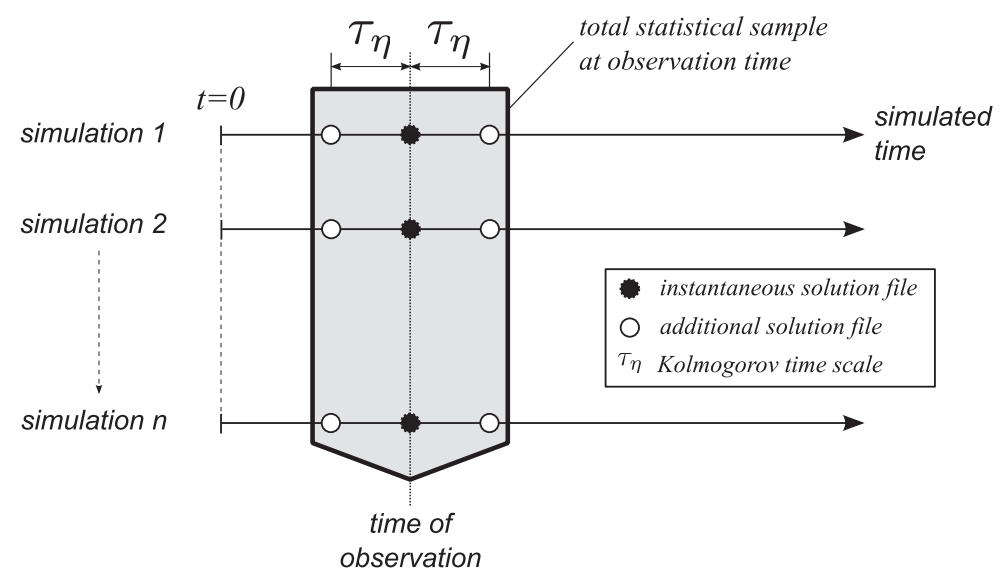

Figure 6. Schematic description of the procedure for evaluating the flow statistics at a given time of observation for the ablative surface simulations.

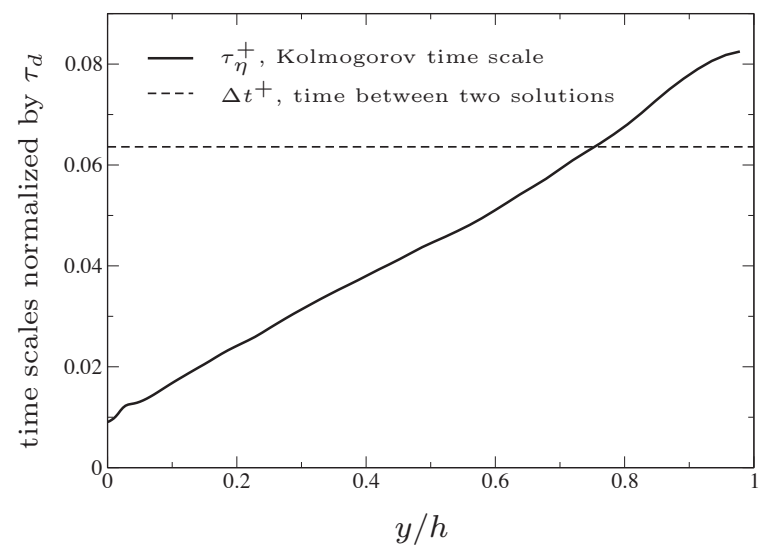

Figure 7. Comparison between the wall normal variation of the Kolmogorov time scale and the time that separates two solution files used to fill the statistical sample.

the oxidizing species are continuously consumed in the simulated case (which means that no ablation would be observed for an infinite simulated time) whereas in the real case the combustion products passing through the nozzle continuously carry oxidizing species that feed the oxidation mechanism. Hence, the periodic channel configuration, combined with the statistical procedure described above, allows to obtain converged statistics representing the unsteady evolution of the turbulent boundary layer over surface ablation for a given amount of initial oxidizing species. Thereby, in order to analyze the generic behavior of a turbulent boundary layer over ablated surface one must find the appropriate scalings of the different observed variables to render them time independent.

One notably observes in figure 8 that the statistics do not give relevant results before $\tau_{s} \approx \tau_{d}$ which is the necessary time for the initial condition to adapt to the ablation boundary condition. For $\tau_{s}>\tau_{d}$, figure 8 indicates that a linear time evolution properly represents the similar behavior of the Stephan velocity. This result has also been observed for others variables such as the atomic and species concentrations. Indeed, figures 9 and 10 show the time evolution of atomic molar fraction profiles of the carbon atom (whose variation is related to the amount of carbon injected inside the boundary layer by surface ablation) and of the oxygen atom (a relevant quantity for oxidation mechanisms). These figures illustrate that scaling the concentration profiles by their centerline value is appropriate. This result also stands for mass fraction profiles for which the example of water species is 


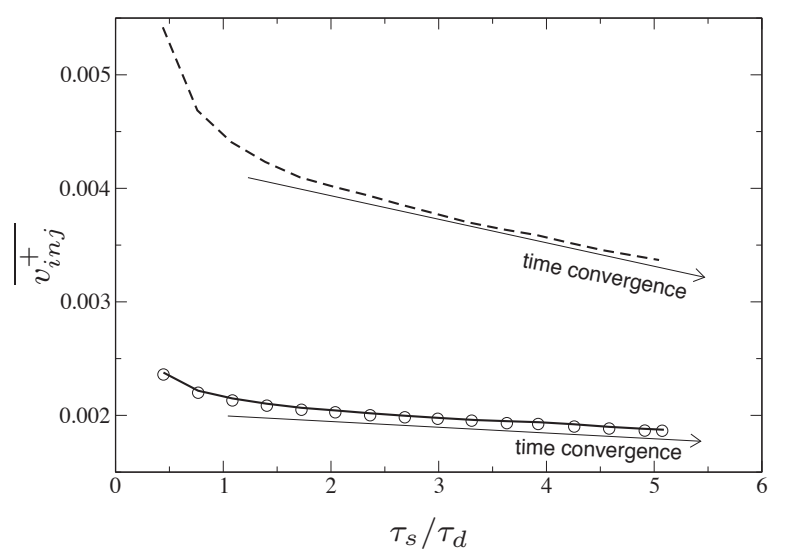

Figure 8. Time convergence of the Stephan velocity expressed in wall units (scaling by $\overline{u_{\tau}}$ ). $\bigcirc$ : case $A 1 ; \quad-$ : case $A 2 ; \quad----$ : case $A 3 ; \quad \rightarrow$ : linear time convergence.
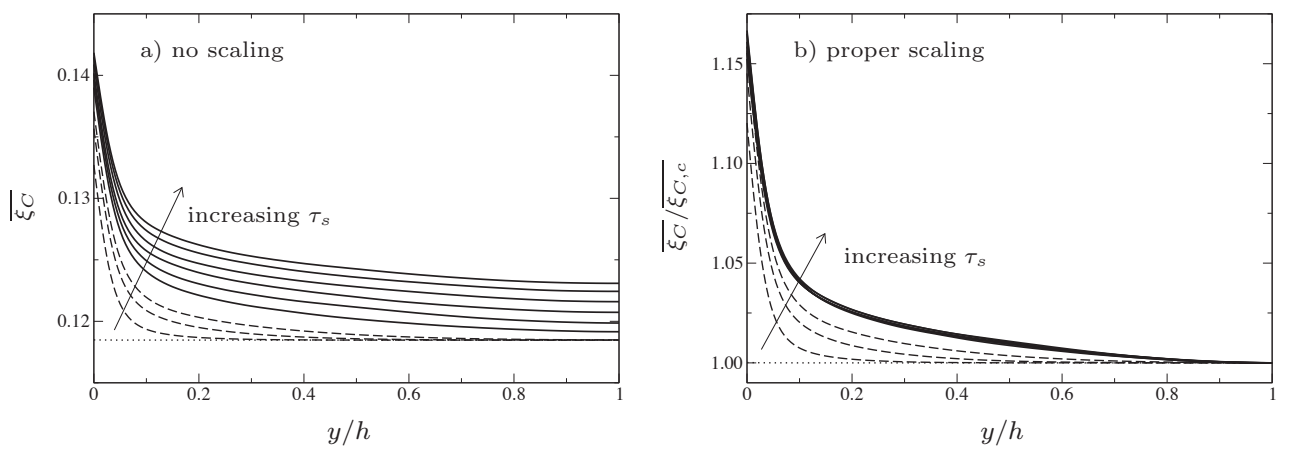

Figure 9. Atomic molar fraction profiles of atom $C$ : a) without scaling; b) scaled by their centerline values. ....... : inert wall (case $I$ ); $\quad----$ : ablative wall simulation (case $A 2$ ) for $\tau_{s}<\tau_{d} ; \quad-\quad$ : ablative wall simulation (case $A 2)$ for $\tau_{s}>\tau_{d}$.
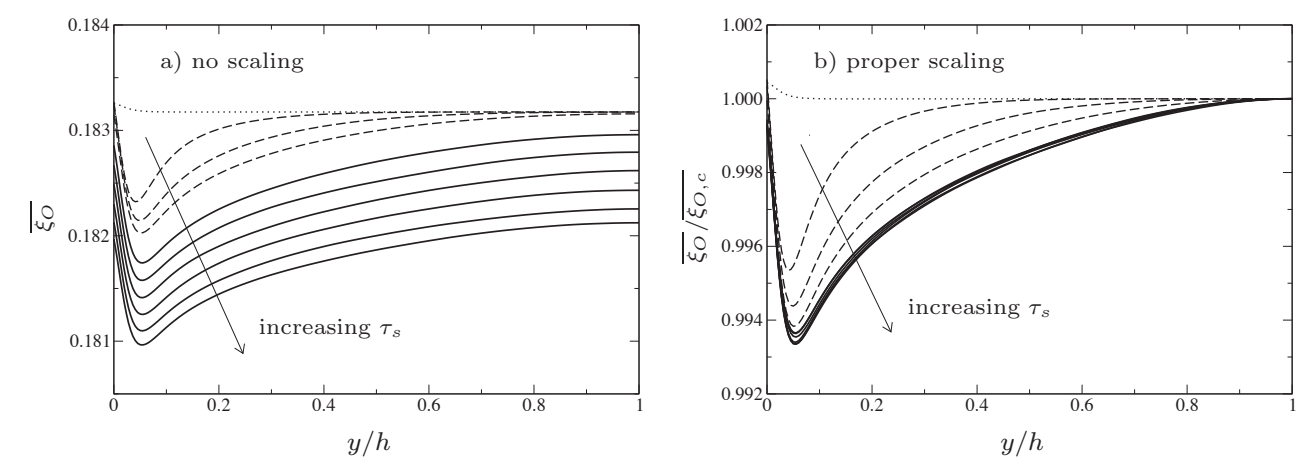

Figure 10. Atomic molar fraction profiles of atom $O$ : a) without scaling; b) scaled by their centerline values. ....... : inert wall (case $I$ ); $\quad----$ : ablative wall simulation (case $A 2$ ) for $\tau_{s}<\tau_{d} ; \quad \longrightarrow$ : ablative wall simulation (case $A 2)$ for $\tau_{s}>\tau_{d}$.

given in figure 11. In figures 9, 10 and 11 the result of the steady inert simulation is also presented to illustrate the strong changes induced by surface ablation. Note that case $A 1$ and $A 3$ are not presented, as well as others atomic and species concentration profiles, because all these data lead to the same conclusion concerning the statistical procedure: for $\tau_{s}>\tau_{d}$, the oxidation mechanism is mainly led by the diffusion of oxidizing species toward the wall and scaling the concentration profiles by their centerline value leads to auto-similar profiles.

Hence $\tau_{d}$ seems to be the correct characteristic time determining the necessary time for the flow to adapt from the initial condition. However, a closer examination 

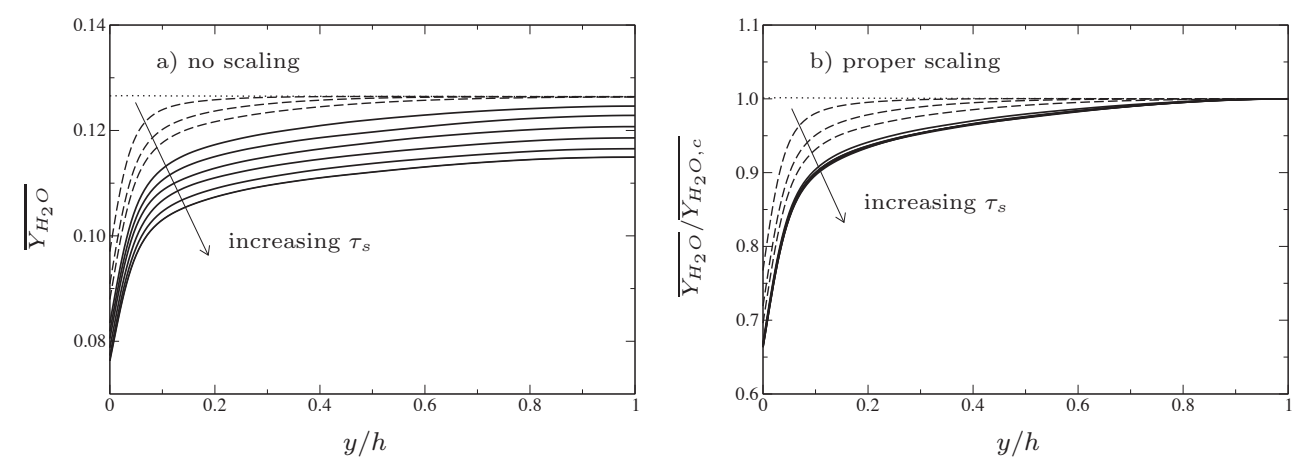

Figure 11. Mass fraction profiles of species $\mathrm{H}_{2} \mathrm{O}$ : a) without scaling; b) scaled by its centerline value. ....... : inert wall (case $I$ ); $\quad----$ : ablative wall simulation (case $A 2$ ) for $\tau_{s}<\tau_{d}$; _ — : ablative wall simulation (case $A 2$ ) for $\tau_{s}>\tau_{d}$.

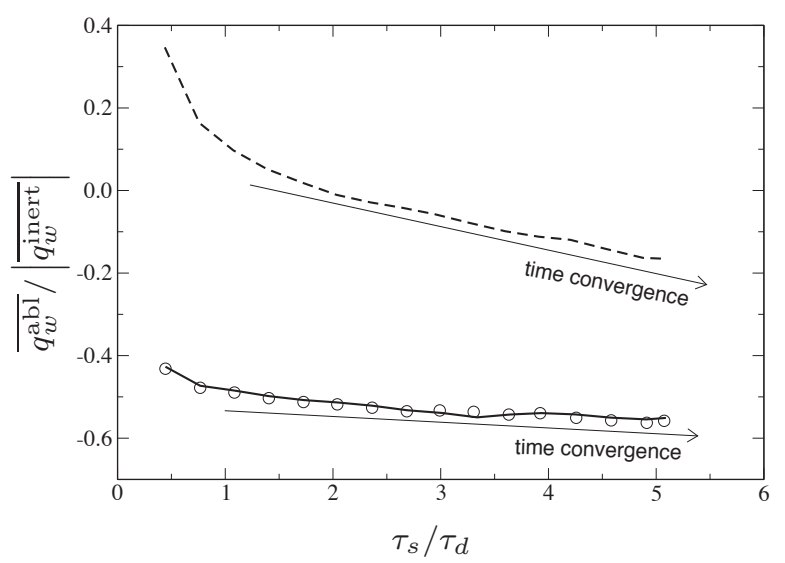

Figure 12. Time convergence of the total wall heat flux for ablative surface simulations, $\overline{q_{w}^{\text {abl }}}$, scaled by the mean modulus of the inert wall heat flux (case $I$ ). $\quad \mathrm{O}$ : case $A 1 ; \quad-\quad$ : case $A 2 ; \quad----$ : case $A 3 ; \quad \rightarrow$ : linear time convergence.

shows that this assessment is too optimistic since the characteristic convergence time can differ when looking at other variables. Considering the whole set of relevant variables, one finds that the wall heat fluxes are the variables which need the longest time to convergence. Indeed, although figure 12 illustrates that the total wall heat flux converges after $\tau_{s}=\tau_{d}$ for all the ablative wall simulations, when all the contributions of the total wall heat flux are analyzed, one shows that the time convergence is only reached for $\tau_{s}>5 \tau_{d}$. This is presented in figure 13 where the time evolution of the Fourier heat flux, $q_{\text {Fourier }}$, the heat flux due to molecular species diffusion, $q_{\mathrm{spec}}$, the sensible enthalpy heat flux, $q_{h_{s}}$, and the chemical enthalpy flux, $q_{h_{c}}$, are plotted with a cumulative representation (the question of heat flux balance is presented further in section 4.3 where the decomposition of each term is clearly explained). All the values are scaled by the modulus of the total wall heat flux presented in figure 12. This explains the sharp peak obtained at around $\tau_{s}=2 \tau_{d}$ for case $A 3$ since the total wall heat flux crosses the zero value for this time of observation.

Finally, the statistics presented hereafter are performed at a simulated time $\tau_{s}=$ $5 \tau_{d}$. This criterion insures a good convergence of the statistics for any variable of interest in the present study. All the ablative wall simulations are thus stopped at about $\tau_{s}=5 \tau_{d}\left(\tau_{s} \approx 20 \tau_{d}\right.$ for the inert wall simulation). This justifies not to take into account the geometrical deformation of the computational domain since during this simulated period of time the relative variation of the channel half-height, $\Delta h / h$, is less than $0.01 \%$. Indeed, considering the most constraining simulation 

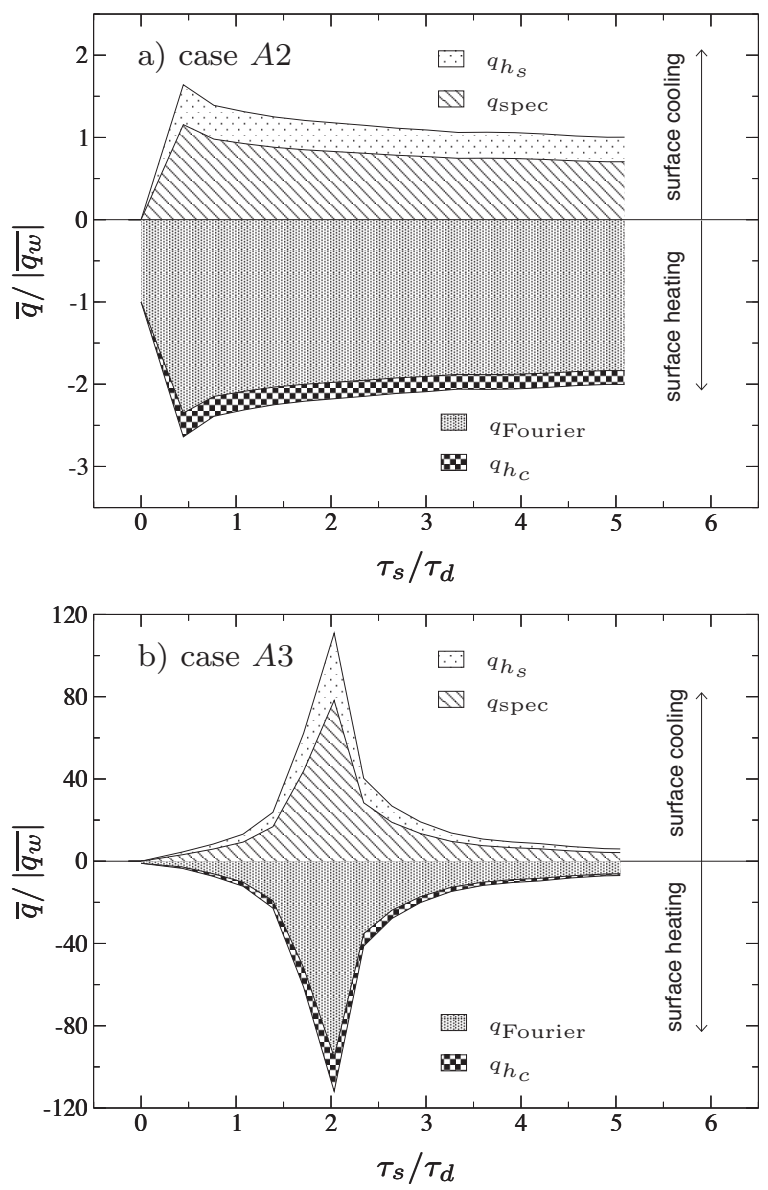

Figure 13. Time convergence of the different wall heat flux contributions during the ablative wall simulations. Heat fluxes are scaled by the mean modulus of the total wall heat flux, $\left|\overline{q_{w}}\right|$. Results of case $A 1$ are not presented because they are very similar to the ones of case $A 2$.

(case $A 3$ ) for which the time averaged injection velocity is about $v_{i n j}^{+} \approx 0.004$, taking $\rho_{c} \approx 1900 \mathrm{~kg} \cdot \mathrm{m}^{-3}$ and $\rho_{w} \approx 7 \mathrm{~kg} \cdot \mathrm{m}^{-3}$, one finds that $\Delta h / \mathrm{h}=\dot{r}_{c} / \tau_{s}=$ $5 v_{i n j}^{+} \rho_{w} / \rho_{c} \approx 0.007 \%$.

\section{Analysis of the flow conservation mechanisms}

\subsection{Species conservation analysis}

We have shown in the previous section 3 that scaling the species mass fraction profiles by their centerline value was an appropriate procedure to analyze species conservation. This is done in figure 14 where the mass fraction profiles of the oxidizing species $\mathrm{H}_{2} \mathrm{O}$ and $\mathrm{CO}_{2}$ are presented so as the species $\mathrm{CO}$ which is relevant to the activity of the global oxidation scheme since this species is produced by both oxidation reactions considered in this study. One first sees in figure 14 that the mean flow is at chemical equilibrium states in all cases (the equilibrium state has been computed a priori thanks to CHEMKIN software specifying the local mean concentrations, pressure and temperature). One recalls that this behavior is not numerically imposed by the code since one makes use of a seven chemical reaction mechanism to describe the flow chemical kinetics. This result is also valid for the other species (not shown herein), which means that the characteristic chemical time scale is negligible compared to the turbulent time scale (i.e. the Damköhler number is high) in the present simulations. 

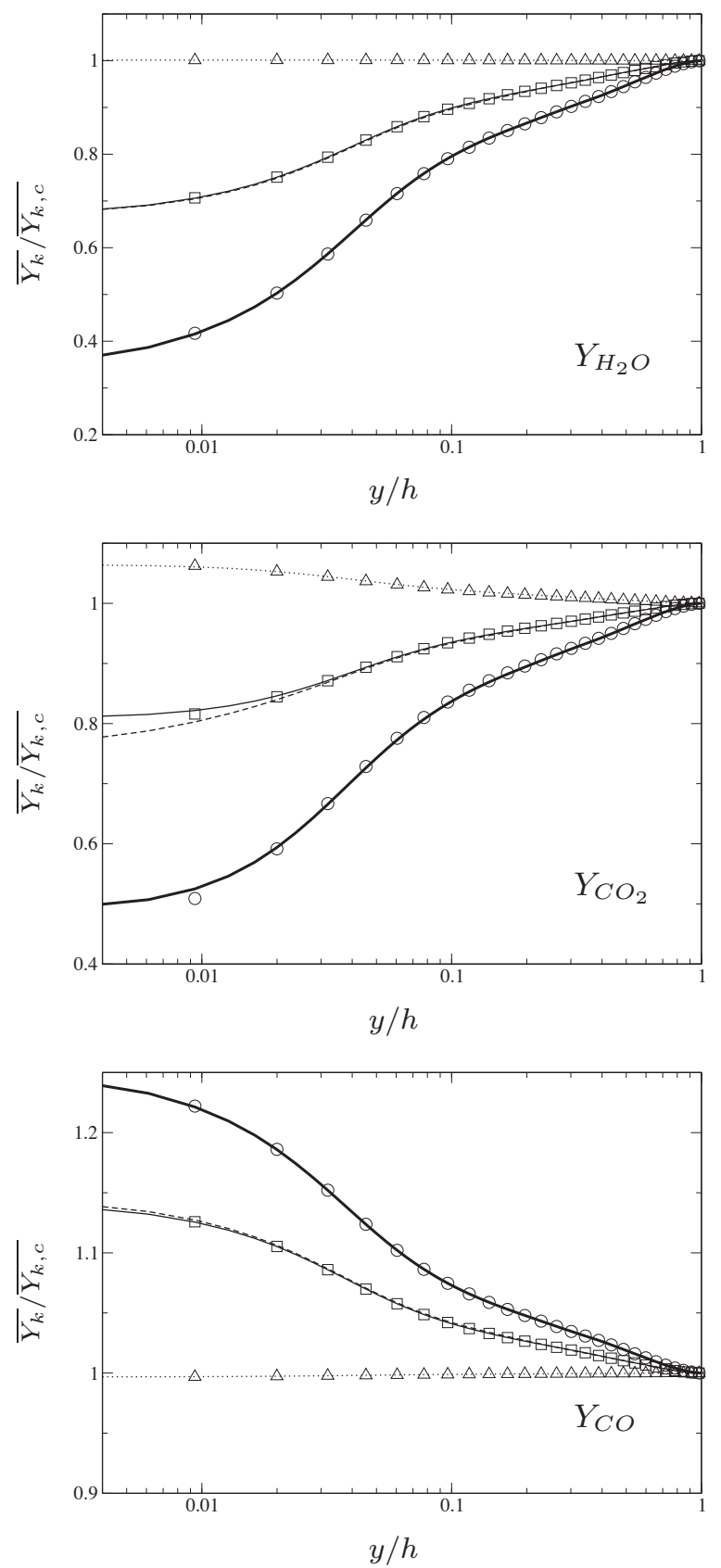

Figure 14. Mean mass fraction profiles of $\mathrm{H}_{2} \mathrm{O}, \mathrm{CO}_{2}$ and $\mathrm{CO}$ species scaled by their centerline value, $\overline{Y_{k, c}}$. Lines represent the DNS results, symbols the equilibrium state computed with CHEMKIN software. The same symbol is used for case $A 1$ and $A 2$ because their equilibrium states are very close. ( $\cdots . . . .$, $\triangle)$ : inert case; $\quad(\square, \square)$ : case $A 1 ; \quad(----, \square)$ : case $A 2 ; \quad(\square, \bigcirc)$ : case $A 3$.

Comparing inert and ablative cases, one also observes that the heterogeneous reactions drastically change the species concentration profiles because of the species consummation/production features of the ablation process: the more oxidation reactions are intense, the more concentration profiles deviate from the inert wall reference case. This is visible in case $A 3$ for which the injection velocity is stronger. Note that the difference between case $A 1$ and $A 2$ are negligible in our simulations. This is mainly due to the fact that the molar concentration of $\mathrm{CO}_{2}$ species at the wall is one order of magnitude less than the one of $\mathrm{H}_{2} \mathrm{O}$. As a consequence, the second oxidation reaction slightly modify the species conservation process. This result is also visible in figures 8 and 12 that clearly indicates no differences in 
the time evolution of the Stephan velocity and the total wall heat flux for the two simulations $A 1$ and $A 2$. Of course one cannot generalize this result since the concentration of oxidizing species at the wall directly depends on the initial concentration delivered by the initial condition. Within the present framework, this means that the only way to control the species concentration at the wall would be to perform another inert simulation changing the operating conditions, and/or the species composition used to initialize the computation. Note however in figure 14 that the flux of $\mathrm{CO}_{2}$ species at the wall (measured by the profile derivative) is not null for case $A 2$ since the consummation of this species is allowed by the oxidation scheme. For all other simulations, this flux is well null which indicates that the simulations behaves properly even if the flow is weakly sensitive to the second oxidation reaction.

\subsection{Momentum conservation balance}

For the inert wall simulation, the average procedure in the homogeneous directions applied to the momentum conservation equation in the streamwise direction leads to the following expression:

$$
\frac{d}{d y} \underbrace{(\underbrace{\overline{\mu d u / d y}}_{\tau_{\text {vis }}} \underbrace{-\bar{\rho} \widetilde{u^{\prime \prime} v^{\prime \prime}}}_{\tau_{\text {tur }}})}_{\tau_{\text {tot }}}=-\overline{\mathcal{S}_{x}}
$$

which differs from the expression obtained for the ablative wall configurations since in these latter cases the time derivative must be conserved, and the non zero injection velocity implies $\widetilde{v} \neq 0$ :

$$
\frac{d}{d y} \underbrace{(\underbrace{\overline{\mu d u / d y}}_{\tau_{\text {vis }}} \underbrace{-\bar{\rho} \widetilde{u^{\prime \prime} v^{\prime \prime}}}_{\tau_{\text {tur }}} \underbrace{-\bar{\rho} \widetilde{u v}}_{\tau_{\text {conv }}})}_{\tau_{\text {tot }}}=-\overline{\mathcal{S}_{x}}+\frac{\partial \bar{\rho} \widetilde{u}}{\partial t}
$$

In equations (31) and (32), $\tau_{\text {vis }}$ stands for the viscous shear stress, $\tau_{\text {tur }}$ the turbulent shear, $\tau_{\text {conv }}$ the convective shear, $\tau_{\text {tot }}$ the total shear, and $\overline{\mathcal{S}_{x}}$ the constant source term that compensates for the streamwise pressure gradient vanishing in periodic channel flow configurations.

Since case $A 3$ is the most constraining regarding to momentum conservation, it has been retained in figure 15 to illustrate the differences between the momentum conservation of inert wall and ablative wall configurations. The -1 slope of the total shear stress for the inert wall simulation (see figure 15-a) is imposed by the source term which is constant in space. This slope is also recovered for the ablative wall simulations (see figure 15-b), which shows that the unsteady term of Eq. (32) is also constant in space. This indicates that the time convergence is verified for this balance, mainly because the unsteady term is less than $1 \%$ of the source term value for all the DNS's, and thus negligible in the balance. Moreover, one observes that the convective term introduced by the ablation process (namely $-\bar{\rho} \widetilde{u} \widetilde{v}$ ) keeps small values. Indeed, the mass flux ratio $F=\overline{\rho_{w} v_{i n j}} / \overline{\rho_{b} u_{b}}$ (b-subscripted variables refering to bulk values) of the current ablative surface simulations is too low compared to classical blowing surface studies[23, 54] to change the shear stress conservation balance: $F \approx 0.01 \%$ for cases $A 1$ and $A 2, F \approx 0.02 \%$ for case $A 3$. The convective term is thus negligible, virtually leading to the same shear stress 

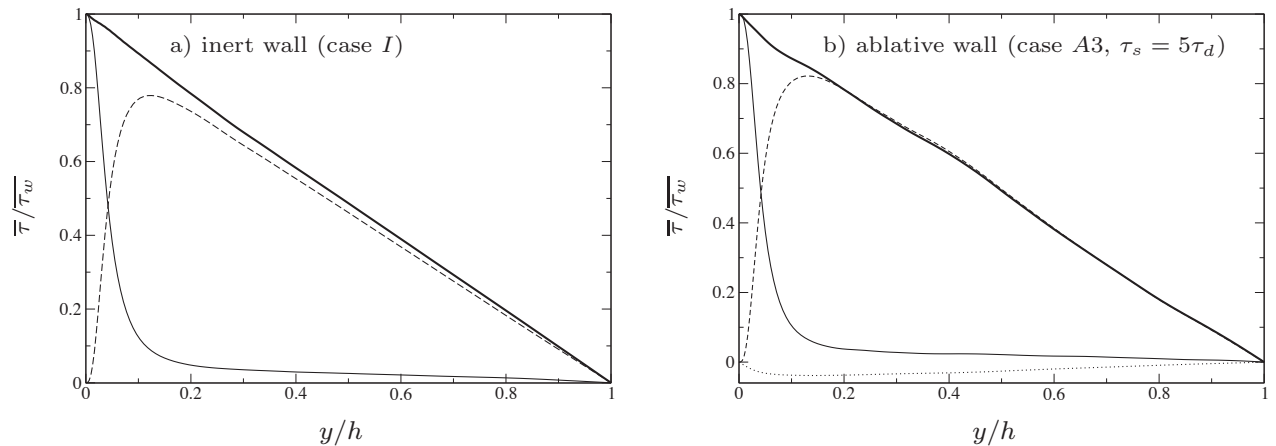

Figure 15. Momentum balance scaled by the mean total shear stress at the wall, $\overline{\tau_{w}}$. a) inert wall case refering to Eq. (31); b) ablative wall case refering to Eq. (32). — — viscous stress, $\tau_{\mathrm{vis}}=\overline{\mu \frac{d u}{d y}}$; ---- : turbulent stress, $\tau_{\text {tur }}=-\bar{\rho} \widetilde{u^{\prime \prime} v^{\prime \prime}} ; \quad \ldots \ldots .$. : convective stress, $\tau_{\text {conv }}=-\bar{\rho} \widetilde{u} \widetilde{v} \quad-\quad-$ : total shear stress, $\tau_{\text {tot. }}$.

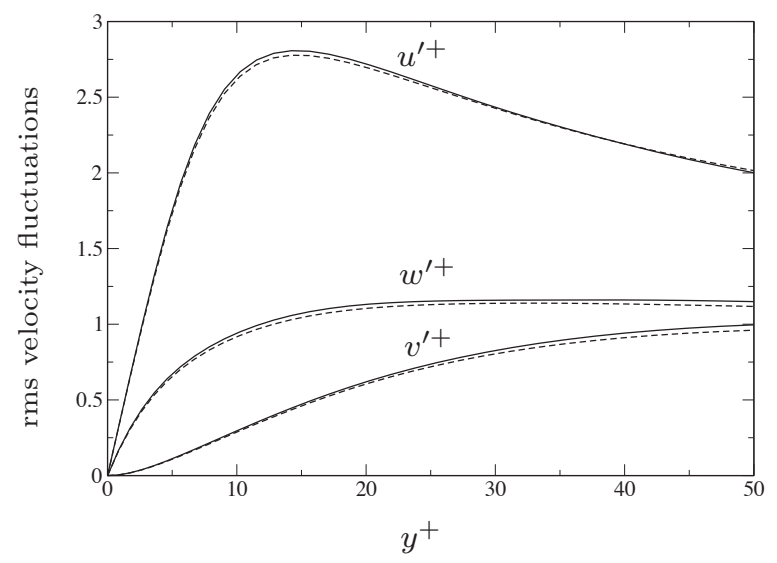

Figure 16. Root-mean-square velocity fluctuations scaled by $\overline{u_{\tau}}$. Comparison between inert wall and ablative wall cases (case $A 2$ and $A 2$ are not shown here because the differences with inert wall simulatio are even less visible). - - - - : inert wall, case $I ; \quad —$ : ablative wall, case $A 3$.

conservation mechanism for both inert and ablative wall turbulent boundary layers. This conclusion is supported by the analysis of the root-mean-square velocity fluctuations presented in figure 16. Indeed, the comparison between the inert case and the most constraining simulation regarding blowing effect (case A3) reveals almost no differences between the two flows. The velocity fluctuations are slightly increased by the surface blowing which has also been reported by Sumitani and Kasagi[23]. However, the present mass flux ratio is about 20 times lower than the one of their study (in which $F \approx 0.4 \%$ ) to observe major differences and notably the displacement of the fluctuation peaks toward the wall.

\subsection{Energy conservation balance}

Neglecting the power of pressure forces and the viscous effect[22], the same analysis procedure is applied to the specific enthalpy conservation equation and leads to the 
following equation for the inert wall configuration:

$$
\frac{d}{d y} \underbrace{(\underbrace{-\overline{\lambda d T / d y}}_{q_{\text {Fourier }}}+\bar{\rho} \underbrace{\left.\bar{\rho} \sum_{k} h_{k} Y_{k} V_{k, y}\right\}}_{q_{\text {spec }}} \underbrace{+\bar{\rho} \widetilde{v^{\prime \prime} h_{s}^{\prime \prime}}}_{q_{h_{s}}}+\underbrace{+\bar{\rho} \sum_{k} \widetilde{v^{\prime \prime} Y_{k}^{\prime \prime}} \Delta h_{f, k}^{0}}_{q_{h_{c}}})}_{q_{\text {tot }}}=\overline{\mathcal{Q}}
$$

and to the following one for ablative surface:

$$
\begin{aligned}
& \frac{d}{d y} \underbrace{(\underbrace{-\overline{\lambda d T / d y}}_{q_{\text {Fourier }}}+\underbrace{\bar{\rho} \sum_{k}\left\{h_{k} Y_{k} V_{k, y}\right\}}_{q_{\text {spec }}} \underbrace{+\bar{\rho}\left(\widetilde{v^{\prime \prime} h_{s}^{\prime \prime}}+\widetilde{v} \widetilde{h_{s}}\right)}_{q_{h_{s}}}+\underbrace{\bar{\rho} \sum_{k}\left(\widetilde{v^{\prime \prime} Y_{k}^{\prime \prime}}+\widetilde{v} \widetilde{Y_{k}}\right) \Delta h_{f, k}^{0}}_{q_{h_{c}}})}_{q_{\text {tot }}} \\
& =\overline{\mathcal{Q}}+\frac{\partial(\bar{p}-\bar{\rho} \tilde{h})}{\partial t}
\end{aligned}
$$

where the operator $\{\cdot\}$ represents a Favre averaged quantity, $q_{h_{s}}$ is the heat flux of sensible enthalpy, $q_{h_{c}}$ the heat flux of chemical enthalpy, $q_{\text {Fourier }}$ the Fourier heat flux, $q_{\text {spec }}$ the heat flux of species diffusion, $\overline{\mathcal{Q}}$ the space constant enthalpy source term that warms the fluid to sustain the mean temperature. Figure 17 presents each term of the total heat flux balance for the inert wall case (figure 17-a), and for ablative wall cases $A 2$ and $A 3$ at $\tau_{s}=5 \tau_{d}$ (figures 17-b and 17-c, respectively). Note that the balances of case $A 1$ is not presented since it is very similar to the one of case $A 2$, leading to the conclusion that the second oxidation reaction has little influence on the heat flux conservation in our simulations. The total heat flux is linear through the boundary layer indicating that the unsteady term of Eq.(34) is constant in space at this time of observation (the unsteady term of Eq. (34) is not negligible in the balance since its value is about $50 \%$ of $\overline{\mathcal{Q}}$ for cases $A 1$ and $A 2$, and about $80 \%$ of $\overline{\mathcal{Q}}$ for case A3). Comparing inert and ablation cases, strong differences are visible notably because of the blowing effect of the ablation process. Indeed, for inert walls the no-slip boundary condition at the wall combined with the continuity equation imposes that $\widetilde{v}=0$. This is not the case for the ablative wall DNS. In addition, the diffusion velocities are not null at the ablative wall. As a consequence, none of the terms of the heat flux balance are null (neither negligible) at the ablative wall whereas the Fourier heat flux is the only contribution in the inert case.

Comparing cases $A 2$ and $A 3$, one observes that the repartition of the fluxes composing the total heat flux are strongly modified depending on the injection velocity. This is also illustrated by table 4 that gives the importance of each heat flux at the wall. This table shows that a stronger injection velocity induces stronger disparities of the fluxes at the wall. Looking at the heat flux balances presented in figure 17 , one finds that the sensible enthalpy and multicomponent fluxes tend to cool the surface, whereas the Fourier and the chemical enthalpy fluxes contribute to surface heating (see also figure 13 where this feature is also visible). The wall surface is globally heated but table 5 indicates that the wall surface would receive a stronger total heat flux if heterogeneous reactions were not present : thanks to 

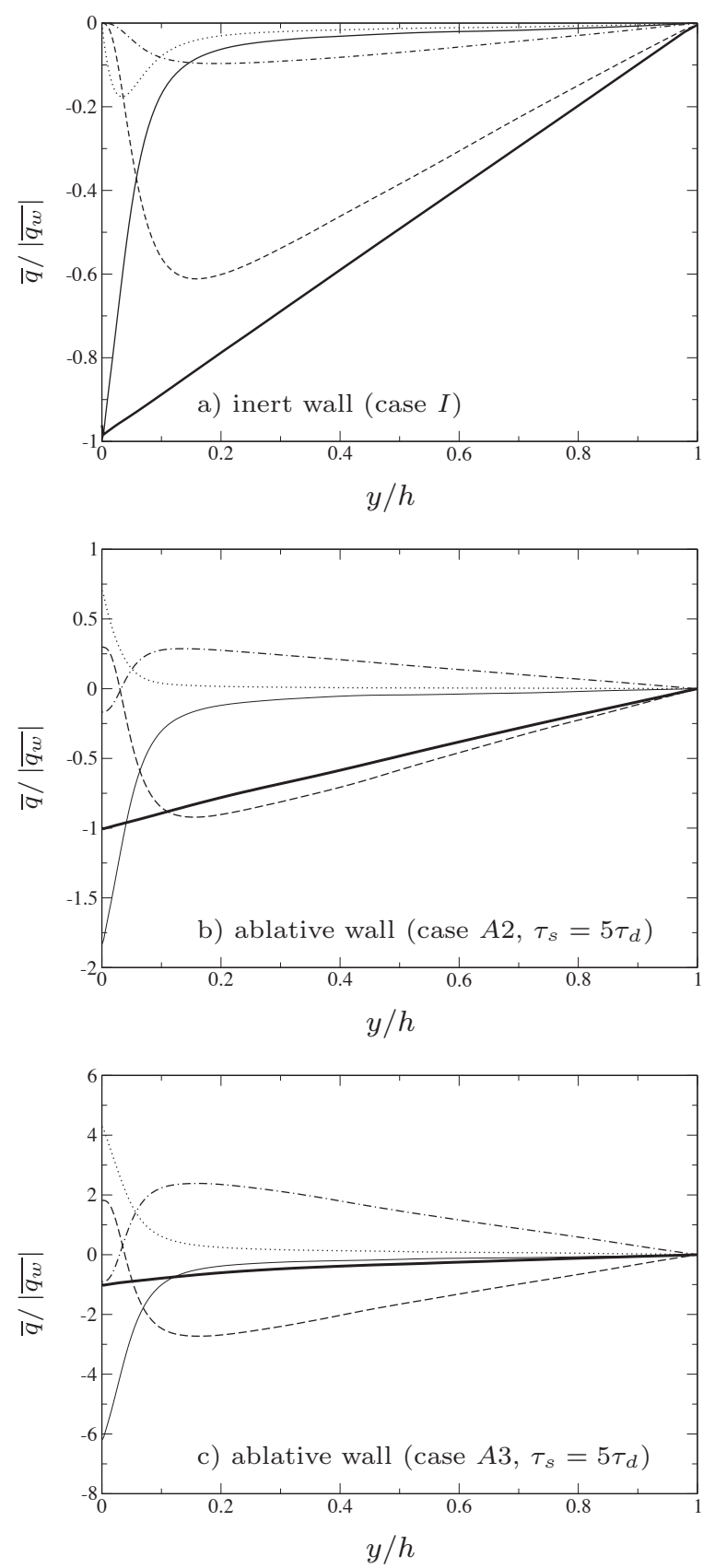

Figure 17. Heat flux balance scaled by the modulus of the flux at the wall $\left|\overline{q_{w}}\right| . \quad----$ : flux of sensible enthalpy, $q_{h_{s}} ; \quad-\quad-$ : flux of chemical enthalpy, $q_{h_{c}} ; \quad-$ : Fourier heat flux, $q_{\text {Fourier }}$; …... : species diffusion flux, $q_{\text {spec }} ; \quad$ : total heat flux, $q_{\text {tot }}$.

surface ablation the total wall heat flux is divided by a factor 1.8 in cases $A 1$ and $A 2$, and by a factor 6.2 for case $A 3$. One also notices in table 5 that the value of the injection velocity directly influences the surface cooling effect of ablation since the total specific enthalpy flux has been divided by a factor 4.3 in case $A 3$, compared to case $A 1$ and $A 2$.

Investigating the case of surface ablation necessarily refers to multicomponent transport modeling, and notably to the questionable role of the Dufour heat flux. Indeed, if the Dufour effect were considered in the molecular heat flux, Eq. (7) 
Table 4. Decomposition of the mean total wall heat flux. Results are scaled by the modulus of the mean total wall heat flux, $\left|\overline{q_{w}}\right|$.

\begin{tabular}{ccccc}
\hline case & $q_{h_{s}} /\left|\overline{q_{w}}\right|$ & $q_{h_{c}} /\left|\overline{q_{w}}\right|$ & $q_{\text {spec }} /\left|\overline{q_{w}}\right|$ & $q_{\text {Fourier }} /\left|\overline{q_{w}}\right|$ \\
\hline inert & $0 \%$ & $0 \%$ & $0 \%$ & $-100 \%$ \\
$A 1$ & $29.2 \%$ & $-16.5 \%$ & $68.1 \%$ & $-180.8 \%$ \\
$A 2$ & $29.8 \%$ & $-16.8 \%$ & $70.4 \%$ & $-183.4 \%$ \\
$A 3$ & $181.9 \%$ & $-90.6 \%$ & $429.2 \%$ & $-620.3 \%$ \\
\hline
\end{tabular}

Table 5. Total wall heat flux of the ablative wall cases scaled by the mean total heat flux of the inert case, $\overline{q_{w}^{\text {inert }}}$.

\begin{tabular}{cc}
\hline case & $\overline{q_{w}^{\text {case }}} / \overline{q_{w}^{\text {inert }}}$ \\
\hline$A 1$ & $56 \%$ \\
$A 2$ & $55 \%$ \\
$A 3$ & $13 \%$ \\
\hline
\end{tabular}

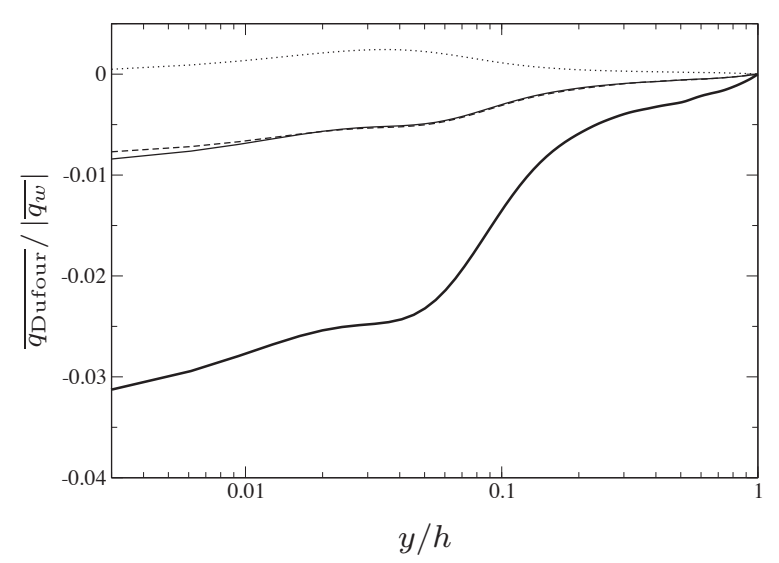

Figure 18. A priori computation of the Dufour heat flux scaled by the modulus of the mean total heat flux at the wall $|\overline{q w}|$. For ablative wall simulations, the results are presented for $\tau_{s}=5 \tau_{d}$. $\quad \cdots \cdots \cdots$ : inert

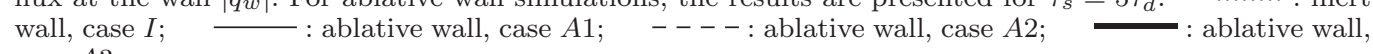
case $A 3$.

would have been replaced by the following one:

$$
q_{i}=\underbrace{-\lambda \frac{\partial T}{\partial x_{i}}}_{\text {Fourier term }}+\underbrace{\rho \sum_{k} h_{k} Y_{k} V_{k, i}}_{\text {species diffusion }}+\underbrace{p \sum_{k} \chi_{k} V_{k, i}}_{\text {Dufour term }}
$$

where $\chi_{k}$ are the species thermal diffusion ratios. To quantify the importance of the Dufour term in the simulations, the EGLIB library[37, 38] has been used to evaluate the thermal diffusion ratios and the Dufour part has been rebuilt a priori using the data of the DNS's for $p$ and $V_{k, i}$. The resulting profile of the wall normal component of the Dufour term, $\overline{q_{\text {Dufour }}}$, has been plotted in figure 18 for all the simulations. Hence, the Dufour heat flux is stronger for the ablative wall cases and notably when the surface oxidation is more intense (see case $A 3$ ). One observes a slight difference between case $A 1$ and $A 2$ in the vicinity of the wall because the species diffusion velocities, proportional to the species concentration wall normal gradients (see Eq. (8)), are not identical since the mass fraction gradient of species $\mathrm{CO}_{2}$ is not null for case $A 2$. This feature has been presented in figure 14 and should also explains the negligible differences observed in tables 4 and 5 between cases $A 1$ and $A 2$. In the region $y / h>0.2$ the Dufour effect clearly has no influence 
whatever the case is, never exceeding $1 \%$ of the total wall heat flux. In the near wall region, its influence is also limited (only $3 \%$ of the total wall heat flux for case $A 3)$. Note that its maximum of influence arises at the wall for ablative wall cases whereas it is displaced just above the wall for inert wall case. Finally, the global analysis of figure 18 allows to conclude that the Dufour effect is negligible for the present boundary layers. However, because the thermal diffusion ratios depends on the mixture composition, this conclusion stands for the present study but would need further examination for any other mixture.

\subsection{Atom conservation balance}

Investigating the conservation mechanism of multicomponent flows is often a hard task because of the number of species conservation equations and of the chemical source terms, $\dot{\omega}_{k}$, that couple the equations all together via the chemical kinetic scheme. For this reason, it can be convenient to investigate the conservation mechanism of atomic elements because no atomic production/consummation arises in the flow itself. In other words, the atomic conservation equations are not coupled to each others because the atomic source term vanishes (the chemical kinetic scheme only redistributes the species concentration while conserving the atomic composition). This type of decoupled variables is often referenced as Shvab-Zel'dovich variables[29]. Hence, the atomic conservation equations can be build starting from the species conservation equation (2), multiplying it by:

$$
M_{a, k}=n_{a, k} \frac{W_{a}}{W_{k}}
$$

where $n_{a, k}$ represents the number of atoms $a$ contained in one species $k$, and $W_{a}$ is the atomic weight of element $a$. Summing over all the species, and applying the ensemble average, it is possible to find an expression for atomic mass fraction conservation in the turbulent boundary layer:

$$
\left.\frac{\partial}{\partial y}(\underbrace{\sum_{k} M_{a, k} \bar{\rho} \widetilde{Y_{k} V_{k, y}}}_{\phi_{\mathrm{vis}, a}}+\underbrace{\bar{\rho}{\widetilde{v^{\prime \prime} \psi_{a}^{\prime \prime}}}^{\bar{\rho}} \widetilde{\rho} \widetilde{v} \widetilde{\psi_{a}}}_{\phi_{\mathrm{tur}, a}})=\overline{\phi_{\text {conv }, a}}\right)=\overline{\dot{\omega}_{a}}
$$

In this expression, $\psi_{a}$ stands for the atomic mass fraction of atom $a$, and $\overline{\dot{\omega}_{a}}=$ $-\partial \bar{\rho} \widetilde{\psi_{a}} / \partial t$ is the source term of atom $a$. One recognizes a classical conservation law where $\phi_{\mathrm{vis}, a}$ is the viscous flux of atom $a, \phi_{\mathrm{tur}, a}$ the turbulent flux, and $\phi_{\mathrm{conv}, a}$ the convective flux. Compared to the classical species conservation equation, this formulation presents the advantage of reducing the number of conservation equations to investigate ( 4 atomic element equations against 7 species equations in the present study).

Hence, the balances of atomic mass fraction fluxes are plotted for each atom in figures 19, 20 and 21, for both inert and ablative wall cases. The case $A 1$ is not presented because it leads to the same conclusions as other ablative wall cases $A 2$ and $A 3$. Concerning the inert wall simulation (figure 19), the atomic conservation process is merely reduced to a balance between the viscous and the turbulent fluxes since the convective term and the atomic source term, $\dot{\omega}_{a}$, are null in this situation. The atomic fluxes for inert wall (figure 19) are scaled by the mean total atomic mass fraction flux of carbon atom at the ablative wall case $A 2, \overline{\phi_{C, w}^{\text {case }}{ }^{2}}$, because 
the flux of carbon atom is null at the wall for the inert case. Moreover, this scaling allows to illustrate that there is approximatively two orders of magnitude between the fluxes of the inert wall case and the ablative wall case $A 2$ which explains that the atomic composition remains constant in the inert wall configuration[22]. This was previously show in figures 9 and 10 .

The structure of the atomic balances for ablative wall cases are very different since the convective term and the source term are both active. From a qualitative point of view, one can observe in figures 20 and 21 that the wall normal variation of the viscous and the turbulent atomic fluxes seem to be of classical type when surface ablation occurs (see the shear stress balance Fig. 15 as a comparison). Note also that normalizing the fluxes by $\overline{\phi_{C, w}}$ seems to be an appropriate scaling to compare the ablative wall cases since the plots of figures 20 and 21 are almost identical. Furthermore, these figures illustrate that the conservation balance of atom $C$ is different from the other ones. The difference is due to the atomic source term which is null for atoms $H, O$, and $N$ (no production or consummation of these atoms can arise in the turbulent boundary layer), whereas the heterogeneous reactions transform the carbon of solid surface into gaseous species containing carbon atoms. The latter process acts like a production source term of carbon atoms injected from the wall surface towards the flow. As a consequence, one observes a -1 slope on the total carbon flux balance which is replaced by a null value for other atoms since no flux of atoms $H, O$, and $N$ can occur in the studied configuration. Note that the constant slope observed for the total atomic flux of atom $C$ indicates that the balances have been performed at a converged time for which the unsteady term of Eq. (37) is constant in space.

Finally, since the turbulent atomic flux is null at the wall, one can verify that the convective and the viscous diffusive fluxes strictly compensate each other at the wall surface for atoms $H, O$, and $N$. Indeed, the heterogeneous reaction mechanism is fed by viscous atomic fluxes, and delivers a convective atomic flux towards the flow. Regarding to carbon atom, one understands that this mechanism is superposed to a convective mechanism ejecting carbon atoms taken from the solid surface towards the boundary layer. The resulting total carbon flux at the wall thus characterizes the solid surface recession.

\section{Conclusions}

This study presents a generic method for performing DNS's of periodic channel flow with ablative walls. A boundary condition for DNS has been developed and used to prescribe the wall normal fluxes of momentum and species induced by the surface heterogeneous reactions. The analysis of the generated data is made easier if the time dependancy can be neglected, which appears to be the case in the present study after a few diffusion times. Making use of ensemble averages to improve the statistical convergence, some particular features of ablative wall turbulent boundary layers have been analyzed such as the chemical equilibrium of the mixture, the effect of the injection velocity on the shear stress and heat flux balances, and the surface cooling effect of wall ablation. It appears that the second surface oxidation reaction (oxidation of carbon material by $\mathrm{CO}_{2}$ species) has no influence on the flow conservation mechanisms and the wall fluxes, mainly because the concentration of $\mathrm{CO}_{2}$ is low in the present simulated mixture. Moreover, the present database allows to study the influence of the Stephan velocity: a higher injection velocity leads to a different repartition of the wall flux contributions, resulting in a more cooled material. However, the tested Stephan velocities, typical of SRM applications, are not strong enough to modify the shear stress conservation mechanism (the convective 

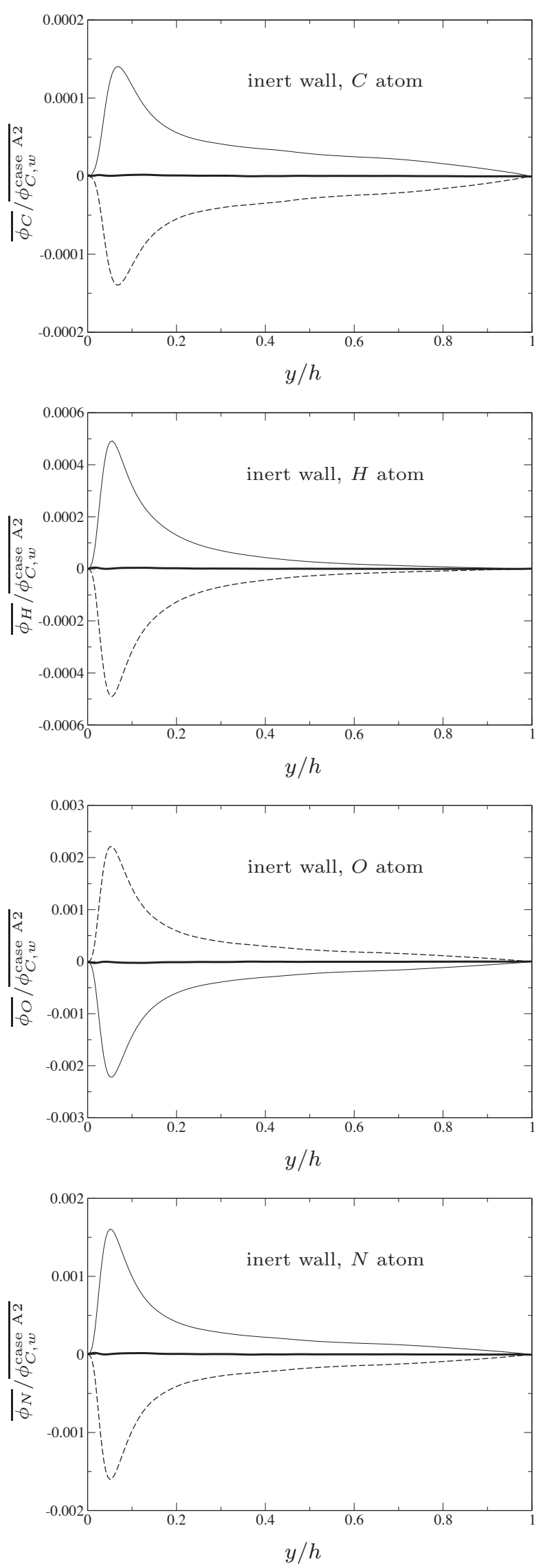

Figure 19. Atomic flux balance for case $I$. Fluxes are scaled by the mean total atomic mass fraction flux of carbon atom at the ablative wall case $A 2, \overline{\phi_{C, w}^{\text {case }}{ }^{2}}$, since $\overline{\phi_{C, w}}$ is null in the inert wall configuration. - : viscous flux, $\phi_{\mathrm{vis}, a} ; \quad----$ : turbulent flux, $\phi_{\mathrm{tur}, a} ; \quad-$ : total atomic flux, $\phi_{\mathrm{tot}, a}=$ $\phi_{\mathrm{vis}, a}+\phi_{\mathrm{tur}, a}$. 

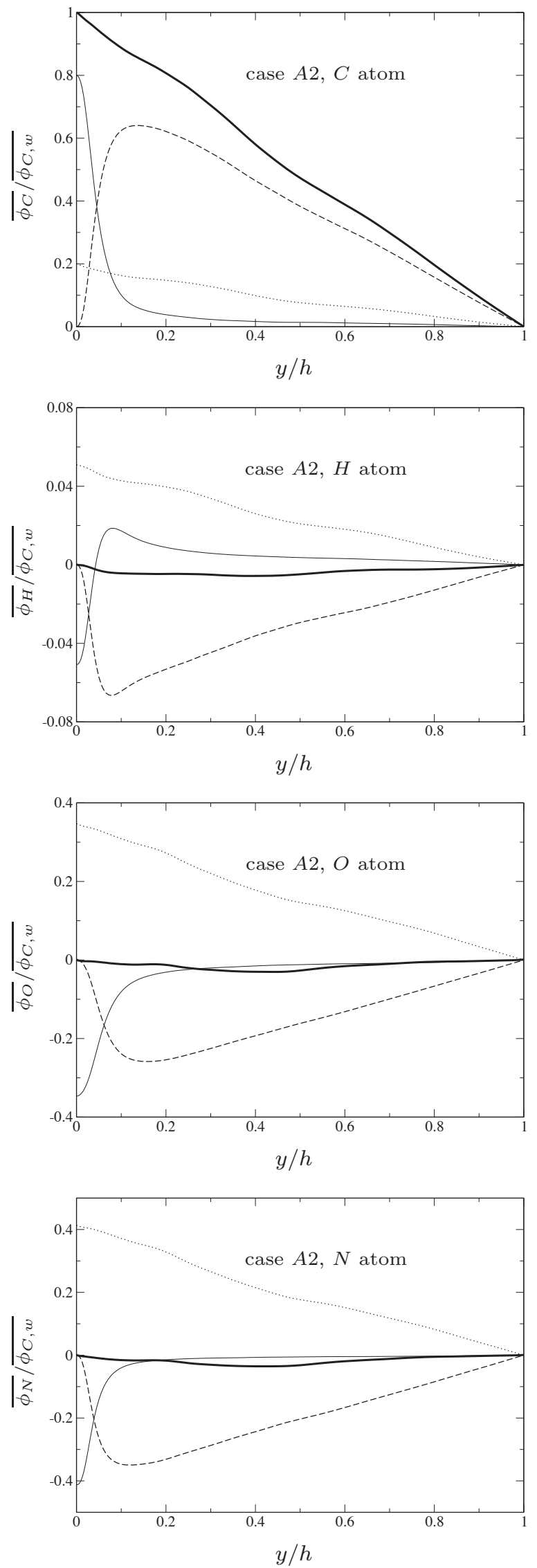

Figure 20. Atomic flux balance for case $A 2$ at $\tau_{s}=5 \tau_{d}$. Fluxes are scaled by the mean total atomic mass fraction flux of carbon atom at the wall $\overline{\phi_{C, w}}$.

$\phi_{\text {tur }, a} ; \quad \cdots \cdots \cdot$ : convective flux, $\phi_{\text {conv }, a}$; : viscous flux, $\phi_{\mathrm{vis}, a} ; \quad----$ : turbulent flux, : total atomic flux, $\phi_{\mathrm{tot}, a}=\phi_{\mathrm{vis}, a}+\phi_{\mathrm{tur}, a}+\phi_{\mathrm{conv}, a}$ 

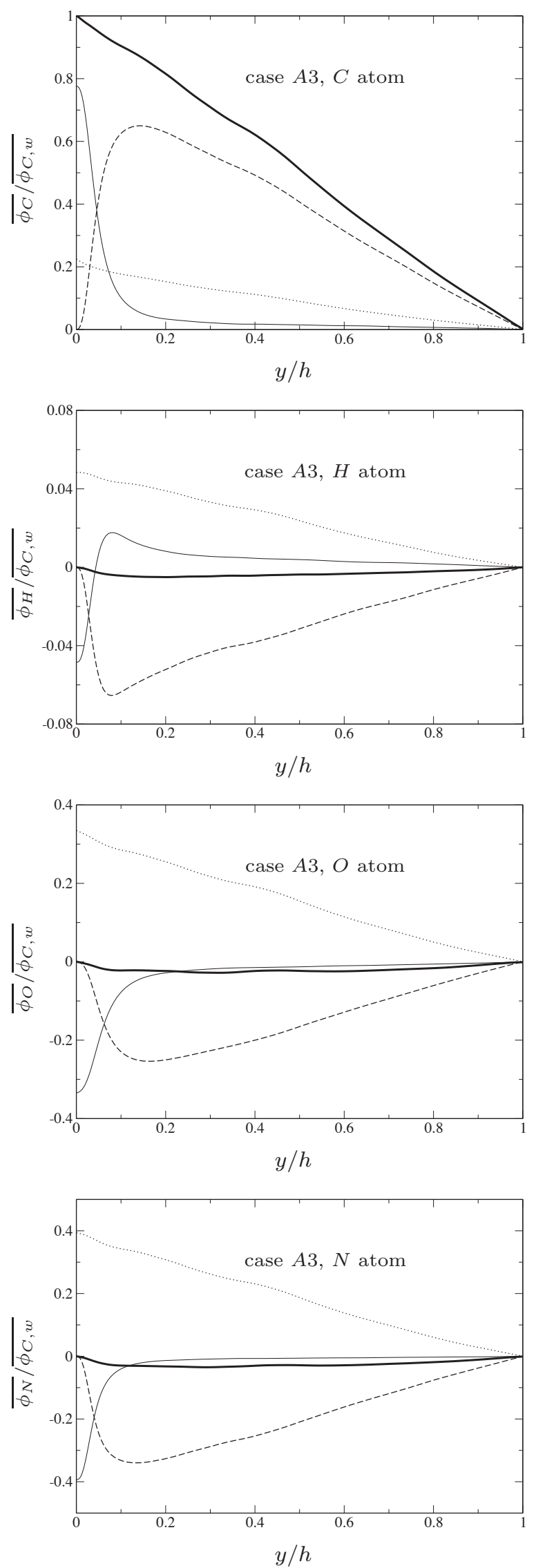

Figure 21. Atomic flux balance for case $\underline{A 3}$ at $\tau_{s}=5 \tau_{d}$. Fluxes are scaled by the mean total atomic mass fraction flux of carbon atom at the wall $\overline{\phi_{C, w}}$. ㄴ. viscous flux, $\phi_{\mathrm{vis}, a} ; \quad---$ : turbulent flux, $\phi_{\text {tur }, a} ; \quad \cdots \cdots \cdot$ : convective flux, $\phi_{\mathrm{conv}, a} ; \quad$ : total atomic flux, $\phi_{\text {tot }, a}=\phi_{\mathrm{vis}, a}+\phi_{\mathrm{tur}, a}+\phi_{\mathrm{conv}, a}$. 
term arising in the momentum conservation equation remains negligible). Finally, an atomic mass fraction conservation equation of Shvab-Zel'dovich type is derived and used to analyze the atomic fluxes in terms of viscous, turbulent and convective contributions. The balances of atomic element fluxes appear to be very similar to the one of a passive scalar.

\section{Acknowledgements}

The authors gratefully acknowledge the CINES for the access to supercomputer facilities, and want to thank the support and expertise of Snecma Propulsion Solide.

\section{References}

[1] Y.K. Chen, and F.S. Milos, Navier-Stokes solutions with finite rate ablation for planetary mission earth reentries, J. Spacecraft and Rockets 42 (2005), pp. 961-970.

[2] J. Zhong, T. Ozawa, and D.A. Levin, Modeling of stardust reentry ablation flows in near-continuum flight regime, AIAA J.46 (2008), pp. 2568-2581.

[3] R.L. Geisler, The Prediction of Graphite Rocket Nozzle Recession Rates, in the 1981 JANNAF Propulsion Meeting, New Orleans, LA, Vol. 5, May, , 1981, pp. 173-196.

[4] B. Evans, P.J. Ferrara, J.D. Moore, and E. Boyd, Evaluation of nozzle erosion characteristics utilizing a rocket motor simulator, AIAA Paper 2006-5245 42nd AIAA/ASME/SAE/ASEE Joint Propulsion Conference \& Exhibit (2006).

[5] P. Thakre, and V. Yang, Chemical erosion of carbon-carbon/graphite nozzles in solid-propellant rocket motors, J. Propulsion and Power 24 (2008), pp. 822-833.

[6] J.H. Koo, D.W.H. Ho, and O.A. Ezekoye, A review of numerical and experimental characterization of thermal protection materials - Part I. numerical modeling, AIAA Paper 2006-4936 42nd AIAA/ASME/SAE/ASEE Joint Propulsion Conference \& Exhibit (2006).

[7] G.L. Vignoles, J. Lachaud, Y. Aspa, and J. Goyhénèche, Ablation of carbon-based materials: multiscale roughness modelling, Composites Sci. and Tech. 69 (2009), pp. 1470-1477.

[8] R.M. Kendall, R.A. Rindal, and E.P. Bartlett, A multicomponent boundary layer chemically coupled to an ablating surface, AIAA J.5 (1967), pp. 1063-1071.

[9] S.T. Keswani, and K.K. Kuo, An aerothermochemical model of carbon-carbon composite nozzle recession, AIAA Paper 83-910 (1983).

[10] T. Cai, and X. Hou, Simple Method for Numerical Simulation of Temperature Response of the Solid Rocket Nozzle, J. Thermophysics 4 (1990), pp. 42-46.

[11] P. Baiocco, and P. Bellomi, A Coupled Thermo-Ablative and Fluid Dynamic Analysis for Numerical Application to Solid Propellant Rockets, AIAA Paper 1996-1811 (1996).

[12] R. Acharya, and K.K. Kuo, Effect of chamber pressure and propellant composition on erosion rate of graphite rocket nozzle, J. Propulsion and Power 23 (2007), pp. 1242-1254.

[13] D. Bianchi, F. Nasuti, and E. Martelli, Coupled analysis of flow and surface ablation in carbon-carbon rocket nozzles, J. Spacecraft and Rockets 46 (2009), pp. 492-500.

[14] J. Kim, P. Moin, and R. Moser, Turbulence statistics in fully developed channel flow at low Reynolds number, J. Fluid Mech. 177 (1987), pp. 133-166.

[15] M. Teitel, and R.A. Antonia, Heat transfer in fully developed turbulent channel flow: comparison between experiment and direct numerical simulations, Int. J. Heat Mass Transfer 36 (1993), pp. 1701-1706.

[16] J.P. Monty, and M.S. Chong, Turbulent channel flow: comparison of streamwise velocity data from experiments and direct numerical simulation, J. Fluid Mech. 633 (2009), pp. 461-474.

[17] S. Hoyas, and J. Jiménez, Scaling the velocity fluctuations in turbulent channel up to Re $e_{\tau}=2003$, Phys. Fluids 18 (2006).

[18] P.G. Huang, G.N. Coleman, and P. Bradshaw, Compressible turbulent channel flows: DNS results and modelling, J. Fluid Mech. 305 (1995), pp. 185-218.

[19] F. Nicoud, Numerical study of a channel flow with variable properties, , in Annual Research Briefs Center for Turbulence Research, NASA Ames/Stanford Univ., 1998, pp. 289-310.

[20] H. Kawamura, H. Abe, and Y. Matsuo, DNS of turbulent heat transfer in channel flow with respect to Reynolds and Prandtl number effects, Int. J. Heat Fluid Flow 20 (1999), pp. 196-207.

[21] Y. Morinishi, S. Tamano, and K. Nakabayashi, Direct numerical simulation of compressible turbulent channel flow between adiabatic and isothermal walls, J. Fluid Mech. 502 (2004), pp. 273-308.

[22] O. Cabrit, and F. Nicoud, Direct simulations for wall modeling of multicomponent reacting compressible turbulent flows, Phys. Fluids 21 (2009), p. 055108.

[23] Y. Sumitani, and N. Kasagi, Direct numerical simulation of turbulent transport with uniform wall injection and suction, AIAA J.33 (1995), pp. 1220-1228.

[24] S. Hahn, J. Je, and H. Choi, Direct numerical simulation of turbulent channel flow with permeable walls, J. Fluid Mech. 450 (2002), pp. 259-285.

[25] A. Velghe, N.T.H. Nguyen-Bui, and P. Chassaing, Direct numerical simulation of reacting turbulent flow on ablatable surface, AIAA Paper 2007-4400 39th AIAA Thermophysics Conference (2007).

[26] V.A. Burakov, and S.F. Sandu, Mathematical Modeling of the Dynamics of Slagging and Thermo- 
chemical Destruction of Carbon Composite Thermal Protective Materials in High-Temperature TwoPhase Flow, Combustion, Explosion, and Shock Waves 33 (1997), pp. 472-481.

[27] H. Wirzberger, and S. Yaniv, Prediction of Erosion in Solid Rocket Motor by Alumina Particles, AIAA Paper 2005-4496 (2005).

[28] K. Klager, The interaction of the efflux of solid propellants with nozzle materials, Propellants, Explosives, Pyrotechnics 2 (1977), pp. 55-63.

[29] F.A. Williams Combustion Theory, 2nd Perseus Books, Reading, Massachusetts, 1985.

[30] K.K. Kuo Principles of combustion, 2nd John Wiley \& Sons, Inc., Hoboken, New Jersey, 2005.

[31] T. Poinsot, and D. Veynante Theoretical and Numerical Combustion, 2nd Edwards, 2005.

[32] J. Amaya, O. Cabrit, D. Poitou, B. Cuenot, and M. El Hafi, Unsteady coupling of Navier-Stokes and radiative heat transfer solvers applied to an anisothermal multicomponent turbulent channel flow, J. Quantitative Spectroscopy \& Radiative Transfer 111 (2010), pp. 295-301.

[33] J. Hirschfelder, F. Curtiss, and R. Bird Molecular theory of gases and liquids, John Wiley \& Sons, 1964.

[34] V. Giovangigli Multicomponent Flow Modeling, Birkhäuser Boston, 1999.

[35] D.A. Cvelbar, Nozzle Recession Study, in the 1981 JANNAF Propulsion Meeting, New Orleans, LA, May, , 1981, pp. 51-68.

[36] S.T. Keswani, E. Andiroglu, J.D. Campbell, and K.K. Kuo, Recession behavior of graphitic nozzles in simulated rocket motors, AIAA Paper 1983-1317 (1983).

[37] A. Ern, and V. Giovangigli Multicomponent transport algorithms, Lecture Notes in Physics, New Series Monographs, m 24 Springer-Verlag, Heidelberg, 1994.

[38] A. Ern, and V. Giovangigli, Fast and accurate multicomponent transport property evaluation, J. Comput. Phys. 120 (1995), pp. 105-116.

[39] R.J. Kee, J.A. Miller, and T.H. Jefferson, Chemkin: A General-Purpose, Problem-independent, Transportable, Fortran Chemical-Kinetics Code Package, SAND80-8003, Sandia National Laboratories, 1980.

[40] R.J. Kee, F.M. Rupley, and J.A. Miller, Chemkin-II: A Fortran Chemical Kinetics Package for the Analysis of Gas-Phase Chemical Kinetics, SAND89-8009B, Sandia National Laboratories, 1989.

[41] A.I. Savvatimskiy, Measurements of the melting point of graphite and the properties of liquid carbon (a review for 1963-2003), Carbon 43 (2005), pp. 1115-1142.

[42] V.R. Gowariker, Mechanical and chemical contributions to the erosion rates of graphite throats in rocket motor nozzles, J. Spacecraft and Rockets 3 (1966), pp. 1490-1494.

[43] H.K. Chelliah, A. Makino, I. Kato, N. Araki, and C.K. Law, Modeling of graphite oxidation in a stagnation-point flow field using detailed homogeneous and semiglobal heterogeneous mechanisms with comparisons to experiments, Combust. Flame 104 (1996), pp. 469-480.

[44] E.C. Golovina, The gasification of carbon by carbon dioxide at high temperatures and pressures, Carbon 18 (1980), pp. 197-201.

[45] P.A. Libby, and T.R. Blake, Theoretical study of burning carbon particles, Combust. Flame 36 (1979), pp. 139-169.

[46] D. Bradley, G. Dixon-Lewis, S. El-Din Habik, and E.M.J. Mushi, The oxidation of graphite powder in flame reaction zones, in 20th Symp. (Int.) on Combustion The Combustion Institute, Pittsburg, 1984, pp. 931-940.

[47] S.T. Keswani, and K.K. Kuo, Validation of an aerothermochemical model for graphite nozzle recession and heat-transfer process, Combust. and Tech. 47 (1986), pp. 177-192.

[48] J. Jiménez, and P. Moin, The minimal flow unit in near-wall turbulence, J. Fluid Mech. 225 (1991), pp. 213-240.

[49] R. Moser, J. Kim, and N. Mansour, Direct numerical simulation of turbulent channel flow up to $R e_{\tau}=590$, Phys. Fluids 11 (1999), pp. 943-945.

[50] V. Moureau, G. Lartigue, Y. Sommerer, C. Angelberger, O. Colin, and T. Poinsot, Numerical methods for unsteady compressible multi-component reacting flows on fixed and moving grids, J. Comput. Phys. 202 (2005), pp. 710-736.

[51] S. Mendez, and F. Nicoud, Large-eddy simulation of a bi-periodic turbulent flow with effusion, J. Fluid Mech. 598 (2008), pp. 27-65.

[52] P. Schmitt, T. Poinsot, B. Schuermans, and K.P. Geigle, Large-eddy simulation and experimental study of heat transfer, nitric oxide emissions and combustion instability in a swirled turbulent highpressure burner, J. Fluid Mech. 570 (2007), pp. 17-46.

[53] O. Colin, and M. Rudgyard, Development of high-order Taylor-Galerkin schemes for unsteady calculations, J. Comput. Phys. 162 (2000), pp. 338-371.

[54] R.L. Simpson, Characteristics of turbulent boundary layers at low Reynolds numbers with and without transpiration, J. Fluid Mech. 42 (1970), pp. 769-802. 